Article

\title{
New Inertial Forward-Backward Mid-Point Methods for Sum of Infinitely Many Accretive Mappings, Variational Inequalities, and Applications
}

\author{
Li Wei ${ }^{1, *}$ (D), Yingzi Shang ${ }^{1}$ and Ravi P. Agarwal ${ }^{2,3}$ \\ 1 School of Mathematics and Statistics, Hebei University of Economics and Business, \\ Shijiazhuang 050061, China; stshangyingzi@heuet.edu.cn \\ 2 Department of Mathematics,Texas A \& M University-Kingsville, Kingsville, TX 78363, USA; \\ Ravi.Agarwal@tamuk.edu \\ 3 Florida Institute of Technology, Melbourne, FL 32901, USA \\ * Correspondence: stweili@heuet.edu.cn or diandianba@yahoo.com
}

Received: 19 April 2019; Accepted: 17 May 2019; Published: 24 May 2019

\begin{abstract}
Some new inertial forward-backward projection iterative algorithms are designed in a real Hilbert space. Under mild assumptions, some strong convergence theorems for common zero points of the sum of two kinds of infinitely many accretive mappings are proved. New projection sets are constructed which provide multiple choices of the iterative sequences. Some already existing iterative algorithms are demonstrated to be special cases of ours. Some inequalities of metric projection and real number sequences are widely used in the proof of the main results. The iterative algorithms have also been modified and extended from pure discussion on the sum of accretive mappings or pure study on variational inequalities to that for both, which complements the previous work. Moreover, the applications of the abstract results on nonlinear capillarity systems are exemplified.
\end{abstract}

Keywords: $\mathrm{m}$-accretive mapping; strongly positive mapping; $\mu$-inversely strongly accretive mapping; $\tau$-Lipschitz continuous mapping; variational inequalities; capillarity systems

MSC: 47H05; 47H09

\section{Introduction and Preliminaries}

Suppose $H$ is a real Hilbert space with norm $\|\cdot\|$ and inner product $\langle\cdot, \cdot\rangle$. Let $K$ be the non-empty closed and convex subset of $H$. We use $\rightarrow$ and $\rightarrow$ to denote the strong and weak convergence in $H$, respectively.

We know that Hilbert space $H$ satisfies Opial's condition in the sense that $\operatorname{limin} f_{n \rightarrow \infty}\left\|x_{n}-z\right\|<$ $\liminf _{n \rightarrow \infty}\left\|x_{n}-y\right\|$ for $\left\{x_{n}\right\} \subset H$ with $x_{n} \rightarrow z$ and $y \neq z$ (see [1]).

The inclusion problem for finding $u \in H$ such that

$$
0 \in S u+T u
$$

is studied intensively, where $S: H \rightarrow H$ is a mapping and $T: H \rightarrow 2^{H}$ is a multi-valued mapping. This is mainly because many problems appear in convex programming, variational inequalities, split feasibility problems, minimization problem, inverse problem and image processing can be modeled by (1).

A mapping $T: D(T) \subset H \rightarrow 2^{H}$ is said to be an accretive mapping (see [2]) if for each $x, y \in D(T)$, there exist $u \in T x$ and $v \in T y$ such that $\langle x-y, u-v\rangle \geq 0$. An accretive mapping $T: D(T) \subset H \rightarrow 2^{H}$ is said to be m-accretive if $R(I+k T)=H$, for $k>0$. 
A mapping $S: D(S) \subset H \rightarrow H$ is said to be $\mu$-inversely strongly accretive mapping (see [3]) if for each $x, y \in D(S)$ and $\mu>0,\langle x-y, S x-S y\rangle \geq \mu\|S x-S y\|^{2}$.

For a mapping $W: D(W) \subset H \rightarrow H$, a point $x \in D(W)$ is called a zero point of $W$ if $W x=0$. The set of zero points of $W$ is denoted by $W^{-1} 0$. If $x \in D(W) \subset H$ satisfies that $W x=x$, then $x$ is called a fixed point of $W$. The set of fixed points of $W$ is denoted by Fix $(W)$.

The study of the special case of inclusion problem (1), where $T$ is accretive and $S$ is $\mu$-inversely strongly accretive, has been a hot topic during the past few years. In particular, the constructions of the iterative algorithms for approximating the zero point of the sum of $T$ and $S$ are focused, see [3-12] and the references therein. The inertial forward-backward splitting method is one of the important iterative algorithms studied by some authors, see [7-9,13,14].

In 2015, Lorenz and Pock [9] proposed the following inertial forward-backward algorithm for approximating zero points of $T+S$, where $T: H \rightarrow 2^{H}$ is m-accretive and $S: H \rightarrow H$ is $\mu$-inversely strongly accretive:

$$
\left\{\begin{array}{l}
u_{0}, u_{1} \in H \text { chosen arbitrarily, } \\
v_{n}=u_{n}+\theta_{n}\left(u_{n}-u_{n-1}\right), \\
u_{n+1}=\left(I+r_{n} T\right)^{-1}\left(v_{n}-r_{n} S v_{n}\right), \quad n \in \mathbb{N} .
\end{array}\right.
$$

In addition, the result that $u_{n} \rightarrow p \in(T+S)^{-1} 0$, as $n \rightarrow \infty$, is proved under some conditions.

To get strong convergence, Dong et al. proposed the following inertial forward-backward projection algorithm in Hilbert spaces in [14]:

$$
\left\{\begin{array}{l}
u_{0}, u_{1} \in H \text { chosen arbitrarily, } \\
v_{n}=u_{n}+\alpha_{n}\left(u_{n}-u_{n-1}\right) \\
w_{n}=\left(I+r_{n} T\right)^{-1}\left(v_{n}-r_{n} S v_{n}\right) \\
\begin{array}{r}
C_{n}=\left\{p \in H:\left\|w_{n}-p\right\|^{2} \leq\left\|u_{n}-p\right\|^{2}-2 \alpha_{n}\left\langle u_{n}-p, u_{n-1}-u_{n}\right\rangle\right. \\
\left.\quad+\alpha_{n}^{2}\left\|u_{n-1}-u_{n}\right\|^{2}\right\}
\end{array} \\
\begin{array}{c}
Q_{n}=\left\{p \in H:\left\langle u_{n}-p, u_{n}-u_{0}\right\rangle \leq 0\right\} \\
u_{n+1}=P_{C_{n} \cap Q_{n}}\left(u_{0}\right), n \in \mathbb{N},
\end{array}
\end{array}\right.
$$

where $T$ and $S$ are the same as those in (2) and $P_{C_{n}} \cap Q_{n}$ is the metric projection whose meaning can be seen in Definition 1 . The projection sets $C_{n}$ and $Q_{n}$ play an important role in the iterative construction to ensure the strong convergence. The result that $u_{n} \rightarrow P_{(T+S)^{-1} 0}\left(u_{0}\right)$, as $n \rightarrow \infty$, is proved under some conditions.

In 2018, Khan et al. proposed the following one in which the projection set $Q_{n}$ is deleted (see [7]):

$$
\left\{\begin{array}{l}
u_{0}, u_{1} \in H \text { chosen arbitrarily, } \\
v_{n}=u_{n}+\theta_{n}\left(u_{n}-u_{n-1}\right), \\
w_{n}=\alpha_{n} u_{n}+\left(1-\alpha_{n}\right)\left(I+r_{n} T\right)^{-1}\left(v_{n}-r_{n} S v_{n}\right), \\
C_{1}:=H, \\
C_{n+1}=\left\{p \in C_{n}:\left\|w_{n}-p\right\|^{2} \leq\left\|u_{n}-p\right\|^{2}\right. \\
\left.\quad-2 \theta_{n}\left(1-\alpha_{n}\right)\left\langle u_{n}-p, u_{n-1}-u_{n}\right\rangle+2 \theta_{n}^{2}\left\|u_{n-1}-u_{n}\right\|^{2}\right\} \\
u_{n+1}=P_{C_{n+1}}\left(u_{0}\right), n \in \mathbb{N},
\end{array}\right.
$$

where $T$ and $S$ are the same as those in (3). The strong convergence that $u_{n} \rightarrow P_{(T+S)^{-1} 0}\left(u_{0}\right)$, as $n \rightarrow \infty$, is also obtained under some conditions.

On the other hand, the inclusion problem (1) is extended to the system of inclusion problems:

$$
0 \in S_{i} u+T_{i} u,
$$


where $T_{i}$ is m-accretive and $S_{i}$ is $\mu_{i}$-inversely strongly accretive for $i \in\{1,2, \cdots, m\}$ or $i \in \mathbb{N}$. In addition, some iterative algorithms for approximating common zero points of $T_{i}+S_{i}$ are constructed in [3,15-17]. In particular, Wei et al. proposed the following implicit mid-point forward-backward projection algorithm in [17]:

$$
\left\{\begin{array}{l}
u_{1} \in H \text { chosen arbitrarily, } \\
w_{n}=\alpha_{n} \eta f\left(u_{n}\right)+\left(I-\alpha_{n} F\right) u_{n} \\
v_{n}=\beta_{n} w_{n}+\delta_{n} \sum_{i=1}^{\infty} c_{n, i}\left(I+r_{n, i} T_{i}\right)^{-1}\left[\frac{w_{n}+v_{n}}{2}-r_{n, i} S_{i}\left(\frac{w_{n}+v_{n}}{2}\right)\right]+\lambda_{n} e_{n} \\
C_{1}=H \\
C_{n+1}=\left\{p \in C_{n}:\left\|v_{n}-p\right\|^{2} \leq \frac{1+\beta_{n}-\lambda_{n}}{2-\delta_{n}}\left\|w_{n}-p\right\|^{2}+\frac{2 \lambda_{n}}{2-\delta_{n}}\left\|e_{n}-p\right\|^{2}\right\} \\
u_{n+1}=P_{C_{n+1}}\left(u_{1}\right), \quad n \in \mathbb{N}
\end{array}\right.
$$

where $f: H \rightarrow H$ is a contraction, $F: H \rightarrow H$ is strongly positive linear bounded mapping, $e_{n}$ is the computational error, $T_{i}$ is m-accretive and $S_{i}$ is $\mu_{i}$-inversely strongly accretive for $i \in \mathbb{N}$. The result that $u_{n} \rightarrow P_{\bigcap_{i=1}^{\infty}\left(T_{i}+S_{i}\right)^{-1} 0}\left(u_{1}\right)$, as $n \rightarrow \infty$, is proved under some conditions.

Recall that $f: H \rightarrow H$ is called a contraction (see [17]) if there exists a constant $l \in(0,1)$ such that $\|f(x)-f(y)\| \leq l\|x-y\|$ for $x, y \in H$.

A mapping $F: H \rightarrow H$ is called strongly positive (see [17]) if there exists $\xi>0$ such that $\langle x, F x\rangle \geq \xi\|x\|^{2}$ for $x \in H$. In this case,

$$
\|a I-b F\|=\sup _{\|x\| \leq 1}|\langle(a I-b F) x, x\rangle|,
$$

where $I$ is the identity mapping, $a \in[0,1]$ and $b \in[-1,1]$.

A mapping $U: H \rightarrow H$ is said to be non-expansive (see [17]) if for each $x, y \in H,\|U x-U y\| \leq$ $\|x-y\|$.

In 2018, Wei et al. proposed some new hybrid iterative algorithms to approximate the common element of the set of zero points of infinitely many m-accretive mappings $T_{i}: H \rightarrow H$ and the set of fixed points of infinitely many non-expansive mappings $B_{i}: H \rightarrow H$. A special case (see Corollary 3.6 in [18]) is presented as follows:

$$
\left\{\begin{array}{l}
u_{1} \in H \text { chosen arbitrarily, } \\
y_{n}=\alpha_{n} u_{n}+\left(1-\alpha_{n}\right) \sum_{i=1}^{\infty} c_{n, i}\left(I+r_{n, i} T_{i}\right)^{-1}\left(u_{n}+e_{n}\right) \\
z_{n}=\beta_{n} u_{n}+\left(1-\beta_{n}\right) \sum_{i=1}^{\infty} b_{i} B_{i} y_{n} \\
C_{1}=H=Q_{1} \\
C_{n+1}=\left\{p \in C_{n}:\left\|y_{n}-p\right\|^{2} \leq \alpha_{n}\left\|u_{n}-p\right\|^{2}+\left(1-\alpha_{n}\right)\left\|u_{n}+e_{n}-p\right\|^{2}\right. \\
\left.\quad\left\|z_{n}-p\right\|^{2} \leq \beta_{n}\left\|u_{n}-p\right\|^{2}+\left(1-\beta_{n}\right)\left\|y_{n}-p\right\|^{2}\right\} \\
Q_{n+1}=\left\{p \in C_{n+1}:\left\|u_{1}-p\right\|^{2} \leq\left\|u_{1}-P_{C_{n+1}}\left(u_{1}\right)\right\|^{2}+\lambda_{n+1}\right\} \\
u_{n+1} \in Q_{n+1}, \quad n \in \mathbb{N}
\end{array}\right.
$$

The result that $u_{n} \rightarrow P_{\bigcap_{m=1}^{\infty}} c_{m}\left(u_{1}\right) \in\left(\bigcap_{i=1}^{\infty} T_{i}^{-1} 0\right) \bigcap\left(\bigcap_{i=1}^{\infty}\right.$ Fix $\left.\left(B_{i}\right)\right)$, as $n \rightarrow \infty$, is proved under some conditions. We may notice that infinite choices of $\left\{u_{n}\right\}$ can be made, which is totally different from traditional projection iterative algorithms, e.g., (3). 
In 2016, Wei et al. proposed an implicit forward-backward mid-point iterative algorithm for approximating common zero points of $T_{i}+S_{i}$, where $T_{i}$ is m-accretive and $S_{i}$ is $\mu_{i}$-inversely strongly accretive, for $i \in \mathbb{N}$. A special case in [19] in the frame of Hilbert space is presented as follows:

$$
\left\{\begin{array}{l}
u_{1} \in K \subset H \text { chosen arbitrarily, } \\
y_{n}=P_{K}\left[\left(1-\alpha_{n}\right)\left(u_{n}+e_{n}^{\prime}\right)\right] \\
z_{n}=\delta_{n} y_{n}+\beta_{n} \sum_{i=1}^{\infty} a_{i}\left(I+r_{n, i} T_{i}\right)^{-1}\left[\left(\frac{y_{n}+z_{n}}{2}-r_{n, i} S_{i}\left(\frac{y_{n}+z_{n}}{2}\right)\right]+\xi_{n} e_{n}^{\prime \prime}\right. \\
u_{n+1}=\gamma_{n} \eta f\left(u_{n}\right)+\left(I-\gamma_{n} F\right) z_{n}+e_{n}^{\prime \prime \prime}, \quad n \in \mathbb{N}
\end{array}\right.
$$

where $f$ and $F$ are the same as those in (6), $\left\{e_{n}^{\prime}\right\},\left\{e_{n}^{\prime \prime}\right\}$ and $\left\{e_{n}^{\prime \prime \prime}\right\}$ are the error sequences. Under some conditions, $\left\{u_{n}\right\}$ is proved to be convergent strongly to $u_{0} \in \bigcap_{i=1}^{\infty}\left(T_{i}+S_{i}\right)^{-1} 0$, which also satisfies the following variational inequality:

$$
\left\langle F u_{0}-\eta f\left(u_{0}\right), u_{0}-z\right\rangle \leq 0, \forall z \in \bigcap_{i=1}^{\infty}\left(T_{i}+S_{i}\right)^{-1} 0 .
$$

We may notice that the connection between the common element of $\left(T_{i}+S_{i}\right)^{-1} 0$ for $i \in \mathbb{N}$ and the solution of one kind variational inequality is set up in [19].

In this paper, our main purpose is formulated as follows: (1) obtain strong convergence theorems instead of weak ones; (2) construct new projection sets, which ensure that infinitely many iterative sequences can be generated compared to traditional projection iterative algorithms (3), (4) and (6); (3) inject the idea of inertial forward-backward algorithm into the iterative construction, compared to iterative algorithms (6)-(8); (4) set up the connection between the common zero point of the sum of two kinds of infinitely many accretive mappings and the solution of one kind variational inequality, which complements the corresponding work since rare studies of the projection iterative algorithms (e.g., (3)-(7)) have mentioned that; (5) provide the application of the abstract result to capillarity systems.

To begin our study, we need some preliminaries.

Definition 1. (see [2]) For the Hilbert space $H$ and its non-empty closed and convex subset $K$, there exists a unique point $x_{0} \in K$ such that $\left\|x-x_{0}\right\|=\inf \{\|x-y\|: y \in K\}$, for each $x \in H$. In this case, the metric projection mapping $P_{K}: H \rightarrow K$ is defined by $P_{K} x=x_{0}$, for $\forall x \in H$.

Definition 2. (see [20]) Let $\left\{K_{n}\right\}$ be a sequence of non-empty closed and convex subsets of $H$. Then

(1) the strong lower limit of $\left\{K_{n}\right\}, s-\operatorname{limin} f K_{n}$, is defined as the set of all $x \in H$ such that there exists $x_{n} \in K_{n}$ for almost all $n$ and it tends to $x$ as $n \rightarrow \infty$ in the norm;

(2) the weak upper limit of $\left\{K_{n}\right\}, w$-limsup $K_{n}$, is defined as the set of all $x \in H$ such that there exists a subsequence $\left\{K_{n_{m}}\right\}$ of $\left\{K_{n}\right\}$ and $x_{n_{m}} \in K_{n_{m}}$ for every $n_{m}$ and it tends to $x$ as $n_{m} \rightarrow \infty$ in the weak topology;

(3) the limit of $\left\{K_{n}\right\}, \lim K_{n}$, is the common value when $s-\liminf K_{n}=w-\limsup K_{n}$.

Lemma 1. (see [20]) Let $\left\{K_{n}\right\}$ be a decreasing sequence of closed and convex subsets of $H$, i.e., $K_{n} \subset K_{m}$ if $n \geq m$. Then $\left\{K_{n}\right\}$ converges in $H$ and $\lim K_{n}=\bigcap_{n=1}^{\infty} K_{n}$.

Lemma 2. (see [21]) Suppose $H$ is a real Hilbert space. If lim $K_{n}$ exists and is not empty, then $P_{K_{n}} x \rightarrow P_{\text {lim } K_{n}} x$ for every $x \in H$, as $n \rightarrow \infty$.

Lemma 3. (see $[2,19])$ If $B: H \rightarrow H$ is accretive, then $(I+r B)^{-1}: H \rightarrow H$ is non-expansive. 
Lemma 4. (see [22]) If $H$ is a real Hilbert space with $K$ its non-empty closed and convex subset, $T_{i}: K \rightarrow$ $K$ is non-expansive for $i \in \mathbb{N}$ and $\sum_{i=1}^{\infty} a_{i}=1$ for $\left\{a_{i}\right\} \subset(0,1)$, then $\sum_{i=1}^{\infty} a_{i} T_{i}$ is non-expansive with $\operatorname{Fix}\left(\sum_{i=1}^{\infty} a_{i} T_{i}\right)=\bigcap_{i=1}^{\infty} \operatorname{Fix}\left(T_{i}\right)$ under the assumption that $\bigcap_{i=1}^{\infty} \operatorname{Fix}\left(T_{i}\right) \neq \varnothing$.

Lemma 5. (see [19]) If $H$ is a real Hilbert space with $K$ its non-empty closed and convex subset, $S: K \rightarrow H$ is a single-valued mapping and $T: H \rightarrow 2^{H}$ is an m-accretive mapping, then

$$
\operatorname{Fix}\left((I+r T)^{-1}(I-r S)\right)=(T+S)^{-1} 0,
$$

for $\forall r>0$.

Lemma 6. (see [23]) Let $H$ be a real Hilbert space and $r \in(0,+\infty)$. Then there exists a continuous, strictly increasing and convex function $g:[0,2 r] \rightarrow[0,+\infty)$ with $g(0)=0$ such that $\|k x+(1-k) y\|^{2} \leq$ $k\|x\|^{2}+(1-k)\|y\|^{2}-k(1-k) g(\|x-y\|)$, for $k \in[0,1], x, y \in H$ with $\|x\| \leq r$ and $\|y\| \leq r$.

Lemma 7. (see [24]) Let $K$ be the non-empty closed and convex subset of Hilbert space $H$ and $P_{K}: H \rightarrow K$ be the metric projection. Then

(1) for $\forall x \in H$ and $\forall y \in K,\left\|P_{K} x-y\right\|^{2}+\left\|P_{K} x-x\right\|^{2} \leq\|y-x\|^{2}$.

(2) $y=P_{K} x$ if and only if there holds the following inequality $\langle x-y, y-z\rangle \geq 0$, for $\forall z \in K$.

Lemma 8. (see [25]) If $f: H \rightarrow H$ is a contraction, then there is a unique element $x \in H$ such that $f(x)=x$.

\section{Some Inertial Forward-Backward Algorithms}

In this section, unless otherwise stated, we always assume that:

(1) $H$ is a real Hilbert space;

(2) $A_{i}: H \rightarrow H$ is $\mu_{i}$-inversely strongly accretive and $B_{i}: H \rightarrow H$ is m-accretive, for each $i \in \mathbb{N}$. In addition, $\bigcap_{i=1}^{\infty}\left(A_{i}+B_{i}\right)^{-1} 0 \neq \varnothing$;

(3) $\left\{e_{n}\right\} \subset H$ is the computational error;

(4) $\left\{\sigma_{n}\right\},\left\{s_{n, i}\right\}$ and $\left\{\mu_{i}\right\}$ are three real number sequences in $(0,+\infty)$ for $i, n \in \mathbb{N}$;

(5) $\left\{\alpha_{n}\right\},\left\{\beta_{n}\right\}$ and $\left\{\gamma_{n}\right\}$ are three real number sequences in $(0,1)$ with $\alpha_{n}+\beta_{n}+\gamma_{n} \equiv 1$, for $n \in \mathbb{N}$;

(6) $\left\{\omega_{n, i}\right\}$ is a real number sequence in $(0,1)$ with $\sum_{i=1}^{\infty} \omega_{n, i}=1$, for $n \in \mathbb{N}$;

(7) $\left\{k_{n}\right\}$ is a real number sequence in $[0, k]$ for some $k \in[0,1)$.

\subsection{New Inertial Forward-Backward Projection Algorithms}

Theorem 1. Let $\left\{u_{n}\right\}$ be generated by the following iterative algorithm:

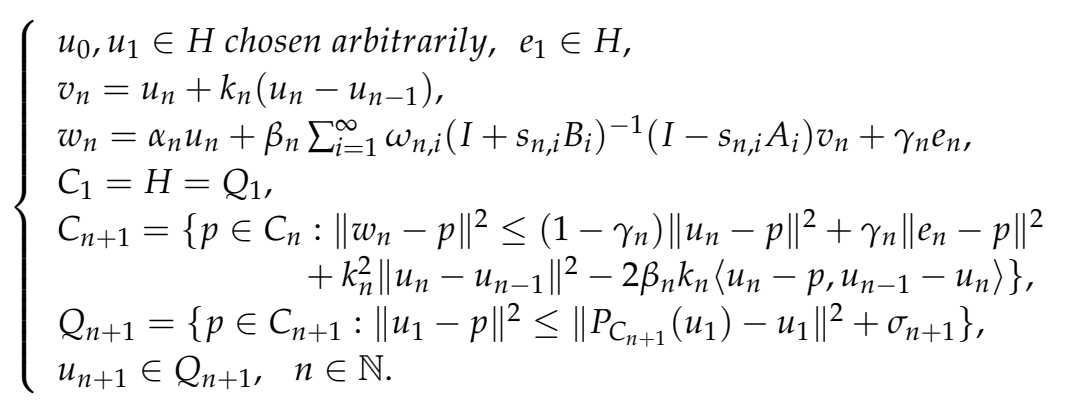

Under the assumptions that: (i) $s_{n, i} \leq \mu_{i}$ for $i, n \in \mathbb{N}$; (ii) $\sigma_{n} \rightarrow 0, \gamma_{n} \rightarrow 0$, as $n \rightarrow \infty$; (iii) $0<\inf _{n} \beta_{n}<1$; (iv) there exists $M>0$ such that $\left\|e_{n}\right\| \leq M$, for $n \in \mathbb{N}$, we have: $u_{n} \rightarrow P_{\cap_{m=1}^{\infty} c_{m}}\left(u_{1}\right)=$ $P_{\bigcap_{i=1}^{\infty}\left(A_{i}+B_{i}\right)^{-1} 0}\left(u_{1}\right) \in \bigcap_{i=1}^{\infty}\left(A_{i}+B_{i}\right)^{-1} 0$, as $n \rightarrow \infty$.

Proof. We split the proof into nine steps. 
Step 1. $\sum_{i=1}^{\infty} \omega_{n, i}\left(I+s_{n, i} B_{i}\right)^{-1}\left(I-s_{n, i} A_{i}\right): H \rightarrow H$ is non-expansive, for $n \in \mathbb{N}$.

The proof of Step 1 is essentially from that of Step 1 in Theorem 2.1 of [17]. For the sake of completeness, we present it as follows.

Since $s_{n, i} \leq 2 \mu_{i}$, then for each $x, y \in H$,

$$
\begin{aligned}
& \left\|\left(I-s_{n, i} A_{i}\right) x-\left(I-s_{n, i} A_{i}\right) y\right\|^{2}=\left\|(x-y)-s_{n, i}\left(A_{i} x-A_{i} y\right)\right\|^{2} \\
& =\|x-y\|^{2}-2 s_{n, i}\left\langle x-y, A_{i} x-A_{i} y\right\rangle+s_{n, i}^{2}\left\|A_{i} x-A_{i} y\right\|^{2} \\
& \leq\|x-y\|^{2}+s_{n, i}\left(s_{n, i}-2 \mu_{i}\right)\left\|A_{i} x-A_{i} y\right\|^{2} \leq\|x-y\|^{2} .
\end{aligned}
$$

Thus, $\left(I-s_{n, i} A_{i}\right): H \rightarrow H$ is non-expansive, for $i, n \in \mathbb{N}$. It then follows from Lemmas 3 and 4 that $\sum_{i=1}^{\infty} \omega_{n, i}\left(I+s_{n, i} B_{i}\right)^{-1}\left(I-s_{n, i} A_{i}\right): H \rightarrow H$ is non-expansive, for $n \in \mathbb{N}$. Combining with Lemma 5, $\bigcap_{i=1}^{\infty} \operatorname{Fix}\left(\left(I+s_{n, i} B_{i}\right)^{-1}\left(I-s_{n, i} A_{i}\right)\right)=\bigcap_{i=1}^{\infty}\left(A_{i}+B_{i}\right)^{-1} 0$.

Step 2. $\bigcap_{i=1}^{\infty}\left(A_{i}+B_{i}\right)^{-1} 0 \subset C_{n}$, for $n \in \mathbb{N}$.

If $n=1$, it is obvious that $\bigcap_{i=1}^{\infty}\left(A_{i}+B_{i}\right)^{-1} 0 \subset C_{1}$.

Now, $\forall q \in \bigcap_{i=1}^{\infty}\left(A_{i}+B_{i}\right)^{-1} 0$, suppose the result is true for $n=m+1$, then if $n=m+2$, it follows from (10) that

$$
\begin{aligned}
& \left\|v_{m+1}-q\right\|^{2} \\
& =\left\|u_{m+1}-q\right\|^{2}+2 k_{m+1}\left\langle u_{m+1}-q, u_{m+1}-u_{m}\right\rangle+k_{m+1}^{2}\left\|u_{m+1}-u_{m}\right\|^{2} .
\end{aligned}
$$

Using Step 1, we have:

$$
\begin{aligned}
& \left\|w_{m+1}-q\right\|^{2} \\
& \leq \alpha_{m+1}\left\|u_{m+1}-q\right\|^{2}+\beta_{m+1}\left\|v_{m+1}-q\right\|^{2}+\gamma_{m+1}\left\|e_{m+1}-q\right\|^{2} .
\end{aligned}
$$

Combining (11) and (12),

$$
\begin{aligned}
& \left\|w_{m+1}-q\right\|^{2} \leq\left(\alpha_{m+1}+\beta_{m+1}\right)\left\|u_{m+1}-q\right\|^{2} \\
& +\gamma_{m+1}\left\|e_{m+1}-q\right\|^{2}+k_{m+1}^{2}\left\|u_{m+1}-u_{m}\right\|^{2}-2 k_{m+1} \beta_{m+1}\left\langle u_{m+1}-q, u_{m}-u_{m+1}\right\rangle
\end{aligned}
$$

which ensures that $q \in C_{m+2}$. Then by induction, $q \in C_{n}$, for $n \in \mathbb{N}$.

Step 3. $C_{n}$ is a closed and convex subset of $H$, for each $n \in \mathbb{N}$.

It is not difficult to see that

$$
\begin{aligned}
& \left\|w_{n}-p\right\|^{2} \leq\left(1-\gamma_{n}\right)\left\|u_{n}-p\right\|^{2}+\gamma_{n}\left\|e_{n}-p\right\|^{2} \\
& +k_{n}^{2}\left\|u_{n}-u_{n-1}\right\|^{2}-2 \beta_{n} k_{n}\left\langle u_{n}-p, u_{n-1}-u_{n}\right\rangle \\
& \Longleftrightarrow\left\|w_{n}\right\|^{2}-\left(1-\gamma_{n}\right)\left\|u_{n}\right\|^{2}-\gamma_{n}\left\|e_{n}\right\|^{2}-k_{n}^{2}\left\|u_{n}-u_{n-1}\right\|^{2}+2 \beta_{n} k_{n}\left\langle u_{n}, u_{n-1}-u_{n}\right\rangle \\
& \leq 2\left\langle p, w_{n}\right\rangle-2\left(1-\gamma_{n}\right)\left\langle p, u_{n}\right\rangle-2 \gamma_{n}\left\langle p, e_{n}\right\rangle+2 \beta_{n} k_{n}\left\langle p, u_{n-1}-u_{n}\right\rangle .
\end{aligned}
$$

Then $C_{n}$ is a closed and convex subset of $H$, for each $n \in \mathbb{N}$.

Step 4. $Q_{n}$ is non-empty for each $n \in \mathbb{N}$, which ensures that $\left\{u_{n}\right\}$ is well-defined.

From Step 3 and the definition of metric projection, for $\sigma_{n+1}$, there exists $\delta_{n+1} \in C_{n+1}$ such that $\left\|u_{1}-\delta_{n+1}\right\|^{2} \leq\left(\inf _{z \in C_{n+1}}\left\|u_{1}-z\right\|\right)^{2}+\sigma_{n+1}=\left\|P_{C_{n+1}}\left(u_{1}\right)-u_{1}\right\|^{2}+\sigma_{n+1}$. Thus, $Q_{n+1} \neq \varnothing$, for $n \in \mathbb{N}$. And then $\left\{u_{n}\right\}$ is well-defined.

Step 5. $P_{C_{n+1}}\left(u_{1}\right) \rightarrow P_{\cap_{m=1}^{\infty} C_{m}}\left(u_{1}\right)$, as $n \rightarrow \infty$.

The proof of Step 5 is similar to Step 2 of Theorem 3.1 in [18]. It follows from Lemma 1 that $\lim C_{n}$ exists and $\lim C_{n}=\bigcap_{n=1}^{\infty} C_{n} \neq \varnothing$. Then Lemma 2 implies that $P_{C_{n+1}}\left(u_{1}\right) \rightarrow P_{\bigcap_{m=1}^{\infty} C_{m}}\left(u_{1}\right)$, as $n \rightarrow \infty$.

Step 6. $u_{n} \rightarrow P_{\cap_{m=1}^{\infty}} C_{m}\left(u_{1}\right)$, as $n \rightarrow \infty$.

Since $u_{n+1} \in Q_{n+1} \subset C_{n+1}$ and $C_{n}$ is a convex subset of $H$, then for $\forall t \in(0,1), t P_{C_{n+1}}\left(u_{1}\right)+(1-$ t) $u_{n+1} \in C_{n+1}$, which implies that

$$
\left\|P_{C_{n+1}}\left(u_{1}\right)-u_{1}\right\| \leq\left\|t P_{C_{n+1}}\left(x_{1}\right)+(1-t) u_{n+1}-u_{1}\right\| .
$$


Using Lemma 6, we have

$$
\begin{aligned}
& \left\|t P_{C_{n+1}}\left(u_{1}\right)+(1-t) u_{n+1}-u_{1}\right\|^{2}=\left\|t\left(P_{C_{n+1}}\left(u_{1}\right)-u_{1}\right)+(1-t)\left(u_{n+1}-u_{1}\right)\right\|^{2} \\
& \leq t\left\|P_{C_{n+1}}\left(u_{1}\right)-u_{1}\right\|^{2}+(1-t)\left\|u_{n+1}-u_{1}\right\|^{2}-t(1-t) g\left(\left\|P_{C_{n+1}}\left(u_{1}\right)-u_{n+1}\right\|\right) .
\end{aligned}
$$

Then (13) and (14) ensure that $\operatorname{tg}\left(\left\|P_{C_{n+1}}\left(u_{1}\right)-u_{n+1}\right\|\right) \leq\left\|u_{n+1}-u_{1}\right\|^{2}-\left\|P_{C_{n+1}}\left(u_{1}\right)-u_{1}\right\|^{2} \leq$ $\sigma_{n+1}$. Letting $t \rightarrow 1$ first and then $n \rightarrow \infty$, we know that $P_{C_{n+1}}\left(u_{1}\right)-u_{n+1} \rightarrow 0$ as $n \rightarrow \infty$. From Step 5, $u_{n} \rightarrow P_{\cap_{m=1}^{\infty} C_{m}}\left(u_{1}\right)$, as $n \rightarrow \infty$.

Step 7. $v_{n} \rightarrow P_{\bigcap_{m=1}^{\infty} C_{m}}\left(u_{1}\right)$ and $w_{n} \rightarrow P_{\bigcap_{m=1}^{\infty} C_{m}}\left(u_{1}\right)$, as $n \rightarrow \infty$.

Since $u_{n+1}-u_{n} \rightarrow 0$ and $u_{n+1} \in Q_{n+1} \subset C_{n+1}$, then from (10), $\left\|u_{n+1}-v_{n+1}\right\|=k_{n+1} \| u_{n+1}-$ $u_{n} \| \rightarrow 0$, as $n \rightarrow \infty$. Thus, $v_{n} \rightarrow P_{\bigcap_{m=1}^{\infty}} C_{m}\left(u_{1}\right)$, as $n \rightarrow \infty$. Since $u_{n+1} \in Q_{n+1} \subset C_{n+1}$, then

$$
\begin{aligned}
& \left\|w_{n}-u_{n+1}\right\|^{2} \leq\left(1-\gamma_{n}\right)\left\|u_{n+1}-u_{n}\right\|^{2}+\gamma_{n}\left\|e_{n}-u_{n+1}\right\|^{2} \\
& +k_{n}^{2}\left\|u_{n}-u_{n-1}\right\|^{2}-2 \beta_{n} k_{n}\left\langle u_{n}-u_{n+1}, u_{n-1}-u_{n}\right\rangle \\
& \leq\left(1-\gamma_{n}\right)\left\|u_{n+1}-u_{n}\right\|^{2}+\gamma_{n}\left\|e_{n}-u_{n+1}\right\|^{2}+k_{n}^{2}\left\|u_{n}-u_{n-1}\right\|^{2}+2\left\|u_{n+1}-u_{n}\right\|\left\|u_{n-1}-u_{n}\right\| \rightarrow 0,
\end{aligned}
$$

as $n \rightarrow \infty$. Thus, $w_{n} \rightarrow P_{\bigcap_{m=1}^{\infty} c_{m}}\left(u_{1}\right)$, as $n \rightarrow \infty$.

Step 8. $P_{\bigcap_{m=1}^{\infty} C_{m}}\left(u_{1}\right) \in \bigcap_{i=1}^{\infty}\left(A_{i}+B_{i}\right)^{-1} 0$.

In fact, if, otherwise, $P_{\bigcap_{m=1}^{\infty} c_{m}}\left(u_{1}\right) \bar{\epsilon} \bigcap_{i=1}^{\infty}\left(A_{i}+B_{i}\right)^{-1} 0$. Then $P_{\bigcap_{m=1}^{\infty} c_{m}}\left(u_{1}\right) \neq \sum_{i=1}^{\infty} \omega_{n, i}(I+$ $\left.s_{n, i} B_{i}\right)^{-1}\left(I-s_{n, i} A_{i}\right) P_{\bigcap_{m=1}^{\infty} c_{m}}\left(u_{1}\right)$.

Since $w_{n}=\alpha_{n} u_{n}+\beta_{n} \sum_{i=1}^{\infty} \omega_{n, i}\left(I+s_{n, i} B_{i}\right)^{-1}\left(I-s_{n, i} A_{i}\right) v_{n}+\gamma_{n} e_{n}$, then $\beta_{n}\left[\sum_{i=1}^{\infty} \omega_{n, i}(I+\right.$ $\left.\left.s_{n, i} B_{i}\right)^{-1}\left(I-s_{n, i} A_{i}\right) v_{n}-w_{n}\right]=\alpha_{n}\left(w_{n}-u_{n}\right)+\gamma_{n}\left(w_{n}-e_{n}\right) \rightarrow 0$, as $n \rightarrow \infty$.

Since $\inf _{n} \beta_{n}>0$, then there exists a subsequence of $\{n\}$, which is still denoted by $\{n\}$ such that $\sum_{i=1}^{\infty} \omega_{n, i}\left(I+s_{n, i} B_{i}\right)^{-1}\left(I-s_{n, i} A_{i}\right) v_{n}-w_{n} \rightarrow 0$, as $n \rightarrow \infty$.

Thus, $\sum_{i=1}^{\infty} \omega_{n, i}\left(I+s_{n, i} B_{i}\right)^{-1}\left(I-s_{n, i} A_{i}\right) v_{n} \rightarrow P_{\bigcap_{m=1}^{\infty} c_{m}}\left(u_{1}\right)$, as $n \rightarrow \infty$.

Since $H$ satisfies Opial's condition, then

$$
\begin{aligned}
& \liminf _{n \rightarrow \infty}\left\|v_{n}-P_{\cap_{m=1}^{\infty} c_{m}}\left(u_{1}\right)\right\| \\
& <\liminf _{n \rightarrow \infty}\left\|v_{n}-\sum_{i=1}^{\infty} \omega_{n, i}\left(I+s_{n, i} B_{i}\right)^{-1}\left(I-s_{n, i} A_{i}\right) P_{\bigcap_{m=1}^{\infty} c_{m}}\left(u_{1}\right)\right\| \\
& \leq \liminf _{n \rightarrow \infty}\left\|v_{n}-\sum_{i=1}^{\infty} \omega_{n, i}\left(I+s_{n, i} B_{i}\right)^{-1}\left(I-s_{n, i} A_{i}\right) v_{n}\right\| \\
& +\liminf _{n \rightarrow \infty}\left\|v_{n}-P_{\bigcap_{m=1}^{\infty} c_{m}}\left(u_{1}\right)\right\| \\
& \leq \liminf _{n \rightarrow \infty}\left\|v_{n}-P_{\bigcap_{m=1}^{\infty} c_{m}}\left(u_{1}\right)\right\|,
\end{aligned}
$$

which makes a contradiction! Thus, $P_{\bigcap_{m=1}^{\infty}} C_{m}\left(u_{1}\right) \in \bigcap_{i=1}^{\infty}\left(A_{i}+B_{i}\right)^{-1} 0$.

Step 9. $P_{\cap_{m=1}^{\infty} C_{m}}\left(u_{1}\right)=P_{\bigcap_{i=1}^{\infty}\left(A_{i}+B_{i}\right)^{-1} 0}\left(u_{1}\right)$.

From Step 8, $\left\|P_{\bigcap_{i=1}^{\infty}\left(A_{i}+B_{i}\right)^{-1} 0}\left(u_{1}\right)-u_{1}\right\| \leq\left\|P_{\bigcap_{m=1}^{\infty} C_{m}}\left(u_{1}\right)-u_{1}\right\|$.

On the other hand, since $\bigcap_{i=1}^{\infty}\left(A_{i}+B_{i}\right)^{-1} 0 \subset \bigcap_{m=1}^{\infty} C_{m}$, then $\left\|P_{\bigcap_{m=1}^{\infty} C_{m}}\left(u_{1}\right)-u_{1}\right\| \leq$ $\left\|P_{\bigcap_{i=1}^{\infty}\left(A_{i}+B_{i}\right)^{-1} 0}\left(u_{1}\right)-u_{1}\right\|$. Thus,

$$
\left\|P_{\bigcap_{i=1}^{\infty}\left(A_{i}+B_{i}\right)^{-1} 0}\left(u_{1}\right)-u_{1}\right\|=\left\|P_{\cap_{m=1}^{\infty} C_{m}}\left(u_{1}\right)-u_{1}\right\| .
$$

Using Lemma 7, we have

$$
\begin{aligned}
& \left\|P_{\bigcap_{i=1}^{\infty}\left(A_{i}+B_{i}\right)^{-1} 0}\left(u_{1}\right)-P_{\bigcap_{m=1}^{\infty} C_{m}}\left(u_{1}\right)\right\|^{2}+\left\|P_{\bigcap_{m=1}^{\infty} C_{m}}\left(u_{1}\right)-u_{1}\right\|^{2} \\
& \leq\left\|P_{\bigcap_{i=1}^{\infty}\left(A_{i}+B_{i}\right)^{-1} 0}\left(u_{1}\right)-u_{1}\right\|^{2}=\left\|P_{\bigcap_{m=1}^{\infty} C_{m}}\left(u_{1}\right)-u_{1}\right\|^{2},
\end{aligned}
$$

which implies that $P_{\bigcap_{i=1}^{\infty}\left(A_{i}+B_{i}\right)^{-1} 0}\left(u_{1}\right)=P_{\bigcap_{m=1}^{\infty} C_{m}}\left(u_{1}\right)$.

Remark 1. Compared to (3) and (4), infinitely many m-accretive mappings and infinitely many $\mu_{i}$-inversely strongly accretive mappings are considered in (10). Compared to (6), the idea of inertial forward-backward 
algorithm is embodied in (10). Compared to (3), (4) and (6), infinite choices of the iterative sequences $\left\{u_{n}\right\}$ are defined.

Remark 2. The traditional idea for choosing the unique iterative element $u_{n+1}$ as the metric projection of the initial element in iterative algorithms (3), (4) and (6) is contained in the ideas of (10) in our paper.

In fact, if take $u_{n+1}=P_{C_{n+1}}\left(u_{1}\right)$, we can easily see that

$$
\left\|u_{n+1}-u_{1}\right\|^{2} \leq\left\|P_{C_{n+1}}\left(u_{1}\right)-u_{1}\right\|^{2}+\sigma_{n+1} .
$$

Thus, $u_{n+1}=P_{C_{n+1}}\left(u_{1}\right) \in Q_{n+1}$, which means that this $u_{n+1}$ is a kind of choice of (10).

Corollary 1. If $k_{n} \equiv 0$, then (10) in Theorem 1 becomes to the traditional forward-backward iterative algorithm:

$$
\left\{\begin{array}{l}
u_{0}, u_{1} \in H \text { chosen arbitrarily, } e_{1} \in H \\
w_{n}=\alpha_{n} u_{n}+\beta_{n} \sum_{i=1}^{\infty} \omega_{n, i}\left(I+s_{n, i} B_{i}\right)^{-1}\left(I-s_{n, i} A_{i}\right) u_{n}+\gamma_{n} e_{n}, \\
C_{1}=H=Q_{1}, \\
C_{n+1}=\left\{p \in C_{n}:\left\|w_{n}-p\right\|^{2} \leq\left(1-\gamma_{n}\right)\left\|u_{n}-p\right\|^{2}+\gamma_{n}\left\|e_{n}-p\right\|^{2}\right\} \\
Q_{n+1}=\left\{p \in C_{n+1}:\left\|u_{1}-p\right\|^{2} \leq\left\|P_{C_{n+1}}\left(u_{1}\right)-u_{1}\right\|^{2}+\sigma_{n+1}\right\} \\
u_{n+1} \in Q_{n+1}, \quad n \in \mathbb{N} .
\end{array}\right.
$$

Corollary 2. If $i \equiv 1$, then (10) in Theorem 1 becomes to the following iterative algorithm:

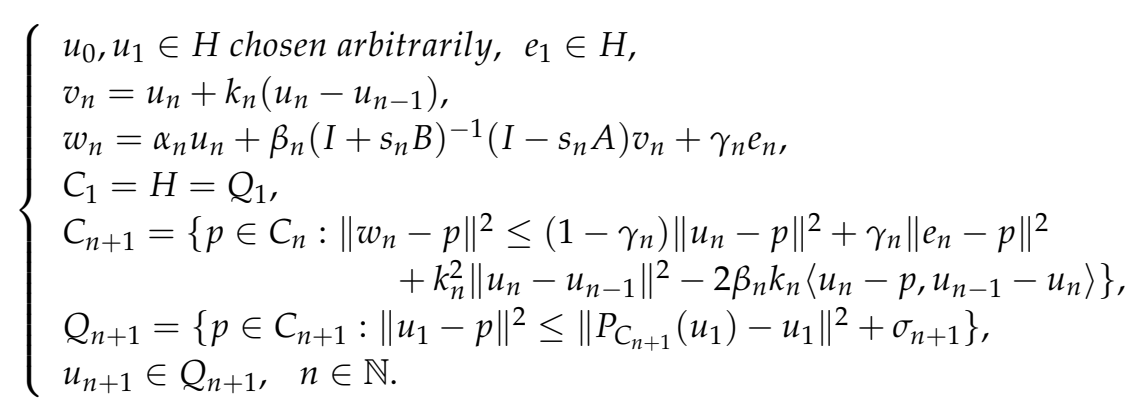

Remark 3. Let $e_{n} \equiv 0, \alpha_{n}+\beta_{n} \equiv 1$, for $n \in \mathbb{N}$. After taking $u_{n+1}=P_{C_{n+1}}\left(u_{1}\right)$ in (15), we may see that $Q_{n+1}$ can be deleted which implies that (15) reduces to (4). However, the strong assumption that $\sum_{n=1}^{\infty} k_{n} \| u_{n}-$ $u_{n-1} \|<+\infty$ in [7] is no longer needed in our paper.

\subsection{New Mid-Point Inertial Forward-Backward Projection Algorithms}

Theorem 2. Suppose $f: H \rightarrow H$ is a contraction with $k \in(0,1)$ and $F: H \rightarrow H$ is a strongly positive linear bounded operator with coefficient $\xi>0$. Let $\left\{u_{n}\right\}$ be generated by the following iterative algorithm:

$$
\left\{\begin{array}{l}
u_{0}, u_{1} \in H \text { chosen arbitrarily, } e_{1} \in H, \\
z_{0}=u_{0}, \\
z_{n}=\delta_{n} \lambda f\left(u_{n}\right)+\left(I-\delta_{n} F\right) u_{n}, \\
v_{n}=z_{n}+k_{n}\left(z_{n}-z_{n-1}\right) \\
w_{n}=\alpha_{n} v_{n}+\beta_{n} \sum_{i=1}^{\infty} \omega_{n, i}\left(I+s_{n, i} B_{i}\right)^{-1}\left(I-s_{n, i} A_{i}\right)\left(\frac{v_{n}+w_{n}}{2}\right)+\gamma_{n} e_{n}, \\
C_{1}=H=Q_{1}, \\
\quad C_{n+1}=\left\{p \in C_{n}:\left\|w_{n}-p\right\|^{2} \leq \frac{2 \alpha_{n}+\beta_{n}}{2-\beta_{n}}\left\|z_{n}-p\right\|^{2}+\frac{2 \gamma_{n}}{2-\beta_{n}}\left\|e_{n}-p\right\|^{2}\right. \\
\left.\quad \quad+\frac{2 \alpha_{n}+\beta_{n}}{2-\beta_{n}} k_{n}^{2}\left\|z_{n}-z_{n-1}\right\|^{2}-2 k_{n} \frac{2 \alpha_{n}+\beta_{n}}{2-\beta_{n}}\left\langle z_{n}-p, z_{n-1}-z_{n}\right\rangle\right\} \\
\quad Q_{n+1}=\left\{p \in C_{n+1}:\left\|u_{1}-p\right\|^{2} \leq\left\|P_{C_{n+1}}\left(u_{1}\right)-u_{1}\right\|^{2}+\sigma_{n+1}\right\} \\
u_{n+1} \in Q_{n+1}, \quad n \in \mathbb{N} .
\end{array}\right.
$$


Under the assumptions of $(i)-(i v)$ in Theorem 1 and $(v) \lambda>0$ and (vi) $\delta_{n} \rightarrow 0$, as $n \rightarrow \infty$, we have: $u_{n} \rightarrow P_{\bigcap_{m=1}^{\infty} C_{m}}\left(u_{1}\right)=P_{\bigcap_{i=1}^{\infty}\left(A_{i}+B_{i}\right)^{-1} 0}\left(u_{1}\right) \in \bigcap_{i=1}^{\infty}\left(A_{i}+B_{i}\right)^{-1} 0$, as $n \rightarrow \infty$.

Proof. We split the proof into ten steps.

Step 1. $\sum_{i=1}^{\infty} \omega_{n, i}\left(I+s_{n, i} B_{i}\right)^{-1}\left(I-s_{n, i} A_{i}\right): H \rightarrow H$ is non-expansive, for $n \in \mathbb{N}$.

Copy Step 1 in Theorem 1.

Step 2. $\left\{w_{n}\right\}$ is well-defined.

Define $U: H \rightarrow H$ by

$$
U x=t y+s T\left(\frac{x+y}{2}\right)+(1-t-s) v,
$$

where $T: H \rightarrow H$ is non-expansive and $t, s \in(0,1)$.

It is easy to check that $U$ is a contraction since

$$
\|U x-U z\| \leq s\left\|\frac{x+y}{2}-\frac{z+y}{2}\right\|=\frac{s}{2}\|x-z\|<\|x-z\|,
$$

for $\forall x, z \in H$.

In view of Lemma 8 , there exists a unique element $x \in H$ such that $x=U x$. Combining with the result of Step 1, $\left\{w_{n}\right\}$ is well-defined.

Step 3. $\cap_{i=1}^{\infty}\left(A_{i}+B_{i}\right)^{-1} 0 \subset C_{n}$, for $n \in \mathbb{N}$.

If $n=1$, it is obvious that $\bigcap_{i=1}^{\infty}\left(A_{i}+B_{i}\right)^{-1} 0 \subset C_{1}$.

Now, $\forall q \in \bigcap_{i=1}^{\infty}\left(A_{i}+B_{i}\right)^{-1} 0$, suppose the result is true for $n=m+1$, then if $n=m+2$, in view of Lemma 4, we have:

$$
\left\|w_{m+1}-q\right\|^{2} \leq \alpha_{m+1}\left\|v_{m+1}-q\right\|^{2}+\beta_{m+1}\left\|\frac{v_{m+1}+w_{m+1}}{2}-q\right\|^{2}+\gamma_{m+1}\left\|e_{m+1}-q\right\|^{2},
$$

which ensures that

$$
\left\|w_{m+1}-q\right\|^{2} \leq \frac{2 \alpha_{m+1}+\beta_{m+1}}{2-\beta_{m+1}}\left\|v_{m+1}-q\right\|^{2}+\frac{2 \gamma_{m+1}}{2-\beta_{m+1}}\left\|e_{m+1}-q\right\|^{2}
$$

It follows from (16) that

$$
\begin{aligned}
& \left\|v_{m+1}-q\right\|^{2} \\
& =\left\|z_{m+1}-q\right\|^{2}+2 k_{m+1}\left\langle z_{m+1}-q, z_{m+1}-z_{m}\right\rangle+k_{m+1}^{2}\left\|z_{m+1}-z_{m}\right\|^{2} .
\end{aligned}
$$

Combining (17) and (18),

$$
\begin{aligned}
& \left\|w_{m+1}-q\right\|^{2} \leq \frac{2 \alpha_{m+1}+\beta_{m+1}}{2-\beta_{m+1}}\left\|z_{m+1}-q\right\|^{2}+\frac{2 \gamma_{m+1}}{2-\beta_{m+1}}\left\|e_{m+1}-q\right\|^{2} \\
& +\frac{2 \alpha_{m+1}+\beta_{m+1}}{2-\beta_{m+1}} k_{m+1}^{2}\left\|z_{m+1}-z_{m}\right\|^{2}-2 k_{m+1} \frac{2 \alpha_{m+1}+\beta_{m+1}}{2-\beta_{m+1}}\left\langle z_{m+1}-q, z_{m}-z_{m+1}\right\rangle .
\end{aligned}
$$

Thus, $q \in C_{m+2}$. Then by induction, $q \in C_{n}$, for $n \in \mathbb{N}$.

Step 4. $C_{n}$ is a closed and convex subset of $H$, for each $n \in \mathbb{N}$. It is not difficult to see that

$$
\begin{aligned}
& \left\|w_{n}-p\right\|^{2} \leq \frac{2 \alpha_{n}+\beta_{n}}{2-\beta_{n}}\left\|z_{n}-p\right\|^{2}+\frac{2 \gamma_{n}}{2-\beta_{n}}\left\|e_{n}-p\right\|^{2} \\
& +k_{n}^{2} \frac{2 \alpha_{n}+\beta_{n}}{2-\beta_{n}}\left\|z_{n}-z_{n-1}\right\|^{2}-2 k_{n} \frac{2 \alpha_{n}+\beta_{n}}{2-\beta_{n}}\left\langle z_{n}-p, z_{n-1}-z_{n}\right\rangle \\
& \Longleftrightarrow\left\|w_{n}\right\|^{2}-\frac{2 \alpha_{n}+\beta_{n}}{2-\beta_{n}}\left\|z_{n}\right\|^{2}-\frac{2 \gamma_{n}}{2-\beta_{n}}\left\|e_{n}\right\|^{2}-k_{n}^{2} \frac{2 \alpha_{n}+\beta_{n}}{2-\beta_{n}}\left\|z_{n}-z_{n-1}\right\|^{2}+2 k_{n} \frac{2 \alpha_{n}+\beta_{n}}{2-\beta_{n}}\left\langle z_{n}, z_{n-1}-z_{n}\right\rangle \\
& \leq 2\left\langle p, w_{n}\right\rangle-2 \frac{2 \alpha_{n}+\beta_{n}}{2-\beta_{n}}\left\langle p, z_{n}\right\rangle-\frac{4 \gamma_{n}}{2-\beta_{n}}\left\langle p, e_{n}\right\rangle+2 k_{n} \frac{2 \alpha_{n}+\beta_{n}}{2-\beta_{n}}\left\langle p, z_{n-1}-z_{n}\right\rangle .
\end{aligned}
$$

Then $C_{n}$ is a closed and convex subset of $H$, for each $n \in \mathbb{N}$.

Step 5. $Q_{n}$ is non-empty for each $n \in \mathbb{N}$, which ensures that $\left\{u_{n}\right\}$ is well-defined.

Copy Step 4 in Theorem 1.

Step 6. $P_{C_{n+1}}\left(u_{1}\right) \rightarrow P_{\cap_{m=1}^{\infty} C_{m}}\left(u_{1}\right)$, as $n \rightarrow \infty$.

Copy Step 5 in Theorem 1. 
Step 7. $u_{n} \rightarrow P_{\cap_{m=1}^{\infty} C_{m}}\left(u_{1}\right)$, as $n \rightarrow \infty$.

Copy Step 6 in Theorem 1.

Step 8. $z_{n} \rightarrow P_{\bigcap_{m=1}^{\infty} c_{m}}\left(u_{1}\right), v_{n} \rightarrow P_{\bigcap_{m=1}^{\infty}} c_{m}\left(u_{1}\right)$ and $w_{n} \rightarrow P_{\bigcap_{m=1}^{\infty} C_{m}}\left(u_{1}\right)$, as $n \rightarrow \infty$.

Since $z_{n}-u_{n}=\delta_{n}\left(\lambda f\left(u_{n}\right)-F u_{n}\right)$ and $\delta_{n} \rightarrow 0$, then it is easy to see that $z_{n} \rightarrow P_{\cap_{m=1}^{\infty} C_{m}}\left(u_{1}\right)$, as $n \rightarrow \infty$.

Since $v_{n}=z_{n}+k_{n}\left(z_{n}-z_{n-1}\right)$, then $v_{n} \rightarrow P_{\cap_{m=1}^{\infty} C_{m}}\left(u_{1}\right)$, as $n \rightarrow \infty$.

Since $u_{n+1}-u_{n} \rightarrow 0$ and $u_{n+1} \in Q_{n+1} \subset C_{n+1}$, then

$$
\begin{aligned}
& \left\|w_{n}-u_{n+1}\right\|^{2} \leq \frac{2 \alpha_{n}+\beta_{n}}{2-\beta_{n}}\left\|z_{n}-u_{n+1}\right\|^{2}+\frac{2 \gamma_{n}}{2-\beta_{n}}\left\|e_{n}-u_{n+1}\right\|^{2} \\
& +k_{n}^{2} \frac{2 \alpha_{n}+\beta_{n}}{2-\beta_{n}}\left\|z_{n}-z_{n-1}\right\|^{2}-2 k_{n} \frac{2 \alpha_{n}+\beta_{n}}{2-\beta_{n}}\left\langle z_{n}-u_{n+1}, z_{n-1}-z_{n}\right\rangle \\
& \leq\left\|z_{n}-u_{n+1}\right\|^{2}+2 \gamma_{n}\left\|e_{n}-z_{n+1}\right\|^{2}+k_{n}^{2}\left\|z_{n}-z_{n-1}\right\|^{2}+2\left\|u_{n+1}-z_{n}\right\|\left\|z_{n-1}-z_{n}\right\| \rightarrow 0 .
\end{aligned}
$$

Thus, $w_{n} \rightarrow P_{\bigcap_{m=1}^{\infty} C_{m}}\left(u_{1}\right)$, as $n \rightarrow \infty$.

Step 9. $P_{\bigcap_{m=1}^{\infty}} C_{m}\left(u_{1}\right) \in \bigcap_{i=1}^{\infty}\left(A_{i}+B_{i}\right)^{-1} 0$.

In fact, if, otherwise, $P_{\bigcap_{m=1}^{\infty} C_{m}}\left(u_{1}\right) \bar{\epsilon} \bigcap_{i=1}^{\infty}\left(A_{i}+B_{i}\right)^{-1} 0$. Then $P_{\bigcap_{m=1}^{\infty} C_{m}}\left(u_{1}\right) \neq \sum_{i=1}^{\infty} \omega_{n, i}(I+$ $\left.s_{n, i} B_{i}\right)^{-1}\left(I-s_{n, i} A_{i}\right) P_{\bigcap_{m=1}^{\infty} C_{m}}\left(u_{1}\right)$.

Since $w_{n}=\alpha_{n} v_{n}+\beta_{n} \sum_{i=1}^{\infty} \omega_{n, i}\left(I+s_{n, i} B_{i}\right)^{-1}\left(I-s_{n, i} A_{i}\right)\left(\frac{v_{n}+w_{n}}{2}\right)+\gamma_{n} e_{n}$, then $\beta_{n}\left[\sum_{i=1}^{\infty} \omega_{n, i}(I+\right.$ $\left.\left.s_{n, i} B_{i}\right)^{-1}\left(I-s_{n, i} A_{i}\right)\left(\frac{v_{n}+w_{n}}{2}\right)-w_{n}\right]=\alpha_{n}\left(w_{n}-v_{n}\right)+\gamma_{n}\left(w_{n}-e_{n}\right) \rightarrow 0$, as $n \rightarrow \infty$.

Since $i n f_{n} \beta_{n}>0$, then there exists a subsequence of $\{n\}$, which is still denoted by $\{n\}$ such that $\sum_{i=1}^{\infty} \omega_{n, i}\left(I+s_{n, i} B_{i}\right)^{-1}\left(I-s_{n, i} A_{i}\right)\left(\frac{v_{n}+w_{n}}{2}\right)-w_{n} \rightarrow 0$, as $n \rightarrow \infty$.

Thus, $\sum_{i=1}^{\infty} \omega_{n, i}\left(I+s_{n, i} B_{i}\right)^{-1}\left(I-s_{n, i} A_{i}\right)\left(\frac{v_{n}+w_{n}}{2}\right) \rightarrow P_{\bigcap_{m=1}^{\infty} C_{m}}\left(u_{1}\right)$, as $n \rightarrow \infty$.

Since $H$ satisfies Opial's condition, then

$$
\begin{aligned}
& \liminf _{n \rightarrow \infty}\left\|\frac{v_{n}+w_{n}}{2}-P_{\cap_{m=1}^{\infty} C_{m}}\left(u_{1}\right)\right\| \\
& <\liminf _{n \rightarrow \infty}\left\|\frac{v_{n}+w_{n}}{2}-\sum_{i=1}^{\infty} \omega_{n, i}\left(I+s_{n, i} B_{i}\right)^{-1}\left(I-s_{n, i} A_{i}\right) P_{\bigcap_{m=1}^{\infty}} c_{m}\left(u_{1}\right)\right\| \\
& \leq \liminf _{n \rightarrow \infty}\left\|\frac{v_{n}+w_{n}}{2}-\sum_{i=1}^{\infty} \omega_{n, i}\left(I+s_{n, i} B_{i}\right)^{-1}\left(I-s_{n, i} A_{i}\right) \frac{v_{n}+w_{n}}{2}\right\| \\
& +\liminf _{n \rightarrow \infty}\left\|\frac{v_{n}+w_{n}}{2}-P_{\bigcap_{m=1}^{\infty} c_{m}}\left(u_{1}\right)\right\| \\
& \leq \liminf _{n \rightarrow \infty}\left\|\frac{\| v_{n}+w_{n}}{2}-P_{\bigcap_{m=1}^{\infty} c_{m}}\left(u_{1}\right)\right\|,
\end{aligned}
$$

which makes a contradiction. Thus, $P_{\bigcap_{m=1}^{\infty} C_{m}}\left(u_{1}\right) \in \bigcap_{i=1}^{\infty}\left(A_{i}+B_{i}\right)^{-1} 0$.

Step 10. $P_{\bigcap_{m=1}^{\infty} C_{m}}\left(u_{1}\right)=P_{\bigcap_{i=1}^{\infty}\left(A_{i}+B_{i}\right)^{-1} 0}\left(u_{1}\right)$.

From Step 9, $\left\|P_{\bigcap_{i=1}^{\infty}\left(A_{i}+B_{i}\right)^{-1} 0}\left(u_{1}\right)-u_{1}\right\| \leq\left\|P_{\bigcap_{m=1}^{\infty}} C_{m}\left(u_{1}\right)-u_{1}\right\|$.

On the other hand, since $\bigcap_{i=1}^{\infty}\left(A_{i}+B_{i}\right)^{-1} 0 \subset \bigcap_{m=1}^{\infty} C_{m}$, then $\left\|P_{\bigcap_{m=1}^{\infty} C_{m}}\left(u_{1}\right)-u_{1}\right\| \leq$ $\left\|P_{\bigcap_{i=1}^{\infty}\left(A_{i}+B_{i}\right)^{-1} 0}\left(u_{1}\right)-u_{1}\right\|$. Thus,

$$
\left\|P_{\bigcap_{i=1}^{\infty}\left(A_{i}+B_{i}\right)^{-1} 0}\left(u_{1}\right)-u_{1}\right\|=\left\|P_{\bigcap_{m=1}^{\infty} C_{m}}\left(u_{1}\right)-u_{1}\right\| .
$$

Using Lemma 7, we have

$$
\begin{aligned}
& \left\|P_{\bigcap_{i=1}^{\infty}\left(A_{i}+B_{i}\right)^{-1} 0}\left(u_{1}\right)-P_{\bigcap_{m=1}^{\infty} C_{m}}\left(u_{1}\right)\right\|^{2}+\left\|P_{\bigcap_{m=1}^{\infty} C_{m}}\left(u_{1}\right)-u_{1}\right\|^{2} \\
& \leq\left\|P_{\bigcap_{i=1}^{\infty}\left(A_{i}+B_{i}\right)^{-1} 0}\left(u_{1}\right)-u_{1}\right\|^{2}=\left\|P_{\bigcap_{m=1}^{\infty} C_{m}}\left(u_{1}\right)-u_{1}\right\|^{2},
\end{aligned}
$$

which implies that $P_{\bigcap_{i=1}^{\infty}\left(A_{i}+B_{i}\right)^{-1} 0}\left(u_{1}\right)=P_{\bigcap_{m=1}^{\infty} C_{m}}\left(u_{1}\right)$.

Remark 4. Similar to Remark $2, u_{n+1}=P_{C_{n+1}}\left(u_{1}\right)$ is also a possible choice of $u_{n+1} \in Q_{n+1}$ in Theorem 2. 
Corollary 3. If $\delta_{n} \equiv 0$, then (16) becomes to the following traditional mid-point inertial forward-backward projection iterative algorithm:

$$
\left\{\begin{array}{l}
u_{0}, u_{1} \in H \text { chosen arbitrarily, } e_{1} \in H \\
v_{n}=u_{n}+k_{n}\left(u_{n}-u_{n-1}\right), \\
w_{n}=\alpha_{n} v_{n}+\beta_{n} \sum_{i=1}^{\infty} \omega_{n, i}\left(I+s_{n, i} B_{i}\right)^{-1}\left(I-s_{n, i} A_{i}\right)\left(\frac{v_{n}+w_{n}}{2}\right)+\gamma_{n} e_{n}, \\
C_{1}=H=Q_{1}, \\
C_{n+1}=\left\{p \in C_{n}:\left\|w_{n}-p\right\|^{2} \leq \frac{2 \alpha_{n}+\beta_{n}}{2-\beta_{n}}\left\|u_{n}-p\right\|^{2}+\frac{2 \gamma_{n}}{2-\beta_{n}}\left\|e_{n}-p\right\|^{2}\right. \\
\left.\quad \quad+\frac{2 \alpha_{n}+\beta_{n}}{2-\beta_{n}} k_{n}^{2}\left\|u_{n}-u_{n-1}\right\|^{2}-2 k_{n} \frac{2 \alpha_{n}+\beta_{n}}{2-\beta_{n}}\left\langle u_{n}-p, u_{n-1}-u_{n}\right\rangle\right\} \\
Q_{n+1}=\left\{p \in C_{n+1}:\left\|u_{1}-p\right\|^{2} \leq\left\|P_{C_{n+1}}\left(u_{1}\right)-u_{1}\right\|^{2}+\sigma_{n+1}\right\} \\
u_{n+1} \in Q_{n+1}, \quad n \in \mathbb{N} .
\end{array}\right.
$$

If, moreover, $k_{n} \equiv 0$ in (19), then it becomes to the following traditional forward-backward mid-point iterative algorithm:

$$
\left\{\begin{array}{l}
u_{0}, u_{1} \in H \text { chosen arbitrarily, } e_{1} \in H \\
w_{n}=\alpha_{n} u_{n}+\beta_{n} \sum_{i=1}^{\infty} \omega_{n, i}\left(I+s_{n, i} B_{i}\right)^{-1}\left(I-s_{n, i} A_{i}\right)\left(\frac{u_{n}+w_{n}}{2}\right)+\gamma_{n} e_{n} \\
C_{1}=H=Q_{1} \\
C_{n+1}=\left\{p \in C_{n}:\left\|w_{n}-p\right\|^{2} \leq \frac{2 \alpha_{n}+\beta_{n}}{2-\beta_{n}}\left\|u_{n}-p\right\|^{2}+\frac{2 \gamma_{n}}{2-\beta_{n}}\left\|e_{n}-p\right\|^{2}\right\} \\
Q_{n+1}=\left\{p \in C_{n+1}:\left\|u_{1}-p\right\|^{2} \leq\left\|P_{C_{n+1}}\left(u_{1}\right)-u_{1}\right\|^{2}+\sigma_{n+1}\right\} \\
u_{n+1} \in Q_{n+1}, \quad n \in \mathbb{N} .
\end{array}\right.
$$

Corollary 4. If $k_{n} \equiv 0$ in (15), then it becomes to the following one:

$$
\left\{\begin{array}{l}
u_{0}, u_{1} \in H \text { chosen arbitrarily, } e_{1} \in H \\
z_{n}=\delta_{n} \lambda f\left(u_{n}\right)+\left(I-\delta_{n} F\right) u_{n} \\
w_{n}=\alpha_{n} z_{n}+\beta_{n} \sum_{i=1}^{\infty} \omega_{n, i}\left(I+s_{n, i} B_{i}\right)^{-1}\left(I-s_{n, i} A_{i}\right)\left(\frac{w_{n}+z_{n}}{2}\right)+\gamma_{n} e_{n} \\
C_{1}=H=Q_{1} \\
C_{n+1}=\left\{p \in C_{n}:\left\|w_{n}-p\right\|^{2} \leq \frac{2 \alpha_{n}+\beta_{n}}{2-\beta_{n}}\left\|z_{n}-p\right\|^{2}+\frac{2 \gamma_{n}}{2-\beta_{n}}\left\|e_{n}-p\right\|^{2}\right\} \\
Q_{n+1}=\left\{p \in C_{n+1}:\left\|u_{1}-p\right\|^{2} \leq\left\|P_{C_{n+1}}\left(u_{1}\right)-u_{1}\right\|^{2}+\sigma_{n+1}\right\} \\
u_{n+1} \in Q_{n+1}, \quad n \in \mathbb{N}
\end{array}\right.
$$

If, moreover, take $u_{n+1}=P_{C_{n+1}}\left(u_{1}\right)$ in (21), then it becomes to (6).

\subsection{Relationship with Variational Inequalities}

A lot work has been done on designing iterative algorithms to approximate solution of variational inequalities due to their wide applications (e.g., [26,27]). A classical variational inequality is to find $u \in K$ such that for $\forall v \in K$,

$$
\langle v-u, T u\rangle \geq 0
$$

where $T: K \rightarrow H$ is a nonlinear mapping. The symbol $V I(K, T)$ denotes the solution of the above variational inequality.

\subsubsection{The First Kind Iteration Theorems}

Definition 3. Let $H$ be a real Hilbert space with $K$ being its non-empty closed and convex subset. $T: K \rightarrow H$ is called a $\tau$-Lipschitz continuous mapping if $\|T x-T y\| \leq \tau\|x-y\|$, for $x, y \in K$. 
Theorem 3. Let $H$ be a real Hilbert space with $K$ being its non-empty closed and convex subset. Suppose $A_{i}$ : $K \rightarrow H$ is $\mu_{i}$-inversely strongly accretive and $B_{i}: K \rightarrow H$ is m-accretive, for each $i \in \mathbb{N}$. Suppose $T: K \rightarrow H$ is an accretive and $\tau$-Lipschitz continuous mapping. Let $\left\{u_{n}\right\}$ be generated by the following iterative algorithm:

$$
\left\{\begin{array}{l}
u_{0} \in K, u_{1} \in K \text { chosen arbitrarily, } e_{1} \in H \\
y_{0}=P_{K}\left(u_{0}-\lambda_{0} T u_{0}\right) \\
y_{n}=P_{K}\left(u_{n}-\lambda_{n} T u_{n}\right) \\
v_{n}=y_{n}+k_{n}\left(y_{n}-y_{n-1}\right) \\
w_{n}=\alpha_{n} v_{n}+\beta_{n} \sum_{i=1}^{\infty} \omega_{n, i}\left(I+s_{n, i} B_{i}\right)^{-1}\left(I-s_{n, i} A_{i}\right) P_{K}\left(u_{n}-\lambda_{n} T y_{n}\right)+\gamma_{n} e_{n} \\
C_{1}=K=Q_{1}, \\
C_{n+1}=\left\{p \in C_{n}:\left\|w_{n}-p\right\|^{2} \leq \alpha_{n}\left\|y_{n}-p\right\|^{2}+\beta_{n}\left\|u_{n}-p\right\|^{2}+\gamma_{n}\left\|e_{n}-p\right\|^{2}\right. \\
\left.\quad+k_{n}^{2}\left\|y_{n}-y_{n-1}\right\|^{2}-2 \alpha_{n} k_{n}\left\langle y_{n}-p, y_{n-1}-y_{n}\right\rangle\right\} \\
Q_{n+1}=\left\{p \in C_{n+1}:\left\|u_{1}-p\right\|^{2} \leq\left\|P_{C_{n+1}}\left(u_{1}\right)-u_{1}\right\|^{2}+\sigma_{n+1}\right\} \\
u_{n+1} \in Q_{n+1}, \quad n \in \mathbb{N} .
\end{array}\right.
$$

Under the assumptions of (i)-(iv) in Theorem 1 and the additional assumptions $(v) \lambda_{n} \rightarrow 0,(v i)$ $\bigcap_{i=1}^{\infty}\left(A_{i}+B_{i}\right)^{-1} 0 \cap \operatorname{VI}(K, T) \neq \varnothing$, we have: $u_{n} \rightarrow P_{\bigcap_{m=1}^{\infty} C_{m}}\left(u_{1}\right)=P_{\bigcap_{i=1}^{\infty}\left(A_{i}+B_{i}\right)^{-1} 0 \cap V I(K, T)}\left(u_{1}\right)$, as $n \rightarrow \infty$.

Proof. We split the proof into nine steps.

Step 1. $\sum_{i=1}^{\infty} \omega_{n, i}\left(I+s_{n, i} B_{i}\right)^{-1}\left(I-s_{n, i} A_{i}\right): K \rightarrow K$ is non-expansive, for $n \in \mathbb{N}$.

Copy the proof of Step 1 in Theorem 1.

Step 2. $\bigcap_{i=1}^{\infty}\left(A_{i}+B_{i}\right)^{-1} 0 \bigcap V I(K, T) \subset C_{n}$, for $n \in \mathbb{N}$.

We first show that $\forall q \in \bigcap_{i=1}^{\infty}\left(A_{i}+B_{i}\right)^{-1} 0 \bigcap V I(K, T)$,

$$
\left\|P_{K}\left(u_{n}-\lambda_{n} T y_{n}\right)-q\right\| \leq\left\|u_{n}-q\right\|
$$

In fact, in view of Lemma 7, we have:

$$
\begin{aligned}
& \left\|P_{K}\left(u_{n}-\lambda_{n} T y_{n}\right)-q\right\|^{2} \\
& \leq\left\|u_{n}-q-\lambda_{n} T y_{n}\right\|^{2}-\left\|u_{n}-P_{K}\left(u_{n}-\lambda_{n} T y_{n}\right)-\lambda_{n} T y_{n}\right\|^{2} \\
& =\left\|u_{n}-q\right\|^{2}-\left\|u_{n}-P_{K}\left(u_{n}-\lambda_{n} T y_{n}\right)\right\|^{2} \\
& +2 \lambda_{n}\left[\left\langle T y_{n}-T q, q-y_{n}\right\rangle+\left\langle T q, q-y_{n}\right\rangle+\left\langle T y_{n}, y_{n}-P_{K}\left(u_{n}-\lambda_{n} T y_{n}\right)\right\rangle\right] \\
& \leq\left\|u_{n}-q\right\|^{2}-\left\|u_{n}-P_{K}\left(u_{n}-\lambda_{n} T y_{n}\right)\right\|^{2}+2 \lambda_{n}\left\langle T y_{n}, y_{n}-P_{K}\left(u_{n}-\lambda_{n} T y_{n}\right)\right\rangle \\
& =\left\|u_{n}-q\right\|^{2}-\left(\left\|u_{n}-y_{n}\right\|^{2}+\left\|y_{n}-P_{K}\left(u_{n}-\lambda_{n} T y_{n}\right)\right\|^{2}\right)+2 \lambda_{n}\left\langle T u_{n}-T y_{n}, P_{K}\left(u_{n}-\lambda_{n} T y_{n}\right)-y_{n}\right\rangle \\
& +2\left\langle u_{n}-y_{n}-\lambda_{n} T u_{n}, P_{K}\left(u_{n}-\lambda_{n} T y_{n}\right)-y_{n}\right\rangle \\
& \leq\left\|u_{n}-q\right\|^{2}-\left(\left\|u_{n}-y_{n}\right\|^{2}+\left\|y_{n}-P_{K}\left(u_{n}-\lambda_{n} T y_{n}\right)\right\|^{2}\right)+2 \lambda_{n} \tau\left\|u_{n}-y_{n}\right\|\left\|y_{n}-P_{K}\left(u_{n}-\lambda_{n} T y_{n}\right)\right\| \\
& \leq\left\|u_{n}-q\right\|^{2}+\left(\lambda_{n}^{2} \tau^{2}-1\right)\left\|u_{n}-y_{n}\right\|^{2} \\
& \leq\left\|u_{n}-q\right\|^{2}
\end{aligned}
$$

which implies that (24) is true.

Next, we can easily check the following by noticing the result of Step 1 and (24):

$$
\begin{aligned}
& \left\|w_{n}-q\right\|^{2} \leq \alpha_{n}\left\|v_{n}-q\right\|^{2}+\beta_{n}\left\|u_{n}-q\right\|^{2}+\gamma_{n}\left\|e_{n}-q\right\|^{2} \\
& \leq \alpha_{n}\left\|y_{n}-q\right\|^{2}+\beta_{n}\left\|u_{n}-q\right\|^{2}+\gamma_{n}\left\|e_{n}-q\right\|^{2}+k_{n}^{2}\left\|y_{n}-y_{n-1}\right\|^{2}-2 \alpha_{n} k_{n}\left\langle y_{n}-q, y_{n-1}-y_{n}\right\rangle .
\end{aligned}
$$

Thus, by induction as that in Theorem $1, q \in C_{n}$, for $n \in \mathbb{N}$.

Step 3. $C_{n}$ is a closed and convex subset of $H$, for each $n \in \mathbb{N}$. 
It is not difficult to see that

$$
\begin{aligned}
& \left\|w_{n}-p\right\|^{2} \leq \alpha_{n}\left\|y_{n}-p\right\|^{2}+\beta_{n}\left\|u_{n}-p\right\|^{2}+\gamma_{n}\left\|e_{n}-p\right\|^{2} \\
& +k_{n}^{2}\left\|y_{n}-y_{n-1}\right\|^{2}-2 \alpha_{n} k_{n}\left\langle y_{n}-p, y_{n-1}-y_{n}\right\rangle \\
& \Longleftrightarrow\left\|w_{n}\right\|^{2}-\alpha_{n}\left\|y_{n}\right\|^{2}-\beta_{n}\left\|u_{n}\right\|^{2}-\gamma_{n}\left\|e_{n}\right\|^{2}-k_{n}^{2}\left\|y_{n}-y_{n-1}\right\|^{2}+2 \alpha_{n} k_{n}\left\langle y_{n}, y_{n-1}-y_{n}\right\rangle \\
& \leq 2\left\langle p, w_{n}\right\rangle-2 \alpha_{n}\left\langle p, y_{n}\right\rangle-2 \beta_{n}\left\langle p, u_{n}\right\rangle-2 \gamma_{n}\left\langle p, e_{n}\right\rangle+2 \alpha_{n} k_{n}\left\langle p, y_{n-1}-y_{n}\right\rangle .
\end{aligned}
$$

Then $C_{n}$ is a closed and convex subset of $H$, for each $n \in \mathbb{N}$.

Copy the results of Steps 4-6 in Theorem 1, we have:

Step 4. $Q_{n}$ is non-empty for each $n \in \mathbb{N}$, which ensures that $\left\{u_{n}\right\}$ is well-defined.

Step 5. $P_{C_{n+1}}\left(u_{1}\right) \rightarrow P_{\cap_{m=1}^{\infty} C_{m}}\left(u_{1}\right)$, as $n \rightarrow \infty$.

Step 6. $u_{n} \rightarrow P_{\cap_{m=1}^{\infty} C_{m}}\left(u_{1}\right)$, as $n \rightarrow \infty$.

Step 7. $y_{n} \rightarrow P_{\bigcap_{m=1}^{\infty}}^{\infty} c_{m}\left(u_{1}\right), v_{n} \rightarrow P_{\bigcap_{m=1}^{\infty}} c_{m}\left(u_{1}\right)$ and $w_{n} \rightarrow P_{\bigcap_{m=1}^{\infty} c_{m}}\left(u_{1}\right)$, as $n \rightarrow \infty$.

It is easy to see that $Q_{n}$ is a closed subset of $K$. Then $u_{n+1} \in Q_{n+1} \subset K$ and $u_{n} \rightarrow P_{\bigcap_{m=1}^{\infty} C_{m}}\left(u_{1}\right)$ imply that $P_{\cap_{m=1}^{\infty}} C_{m}\left(u_{1}\right) \in K$. Therefore,

$$
\begin{aligned}
& \left\|y_{n}-P_{\cap_{m=1}^{\infty} c_{m}}\left(u_{1}\right)\right\| \leq\left\|u_{n}-\lambda_{n} T u_{n}-P_{\cap_{m=1}^{\infty} c_{m}}\left(u_{1}\right)\right\| \\
& \leq\left\|u_{n}-P_{\bigcap_{m=1}^{\infty}} c_{m}\left(u_{1}\right)\right\|+\lambda_{n}\left\|T u_{n}\right\| \rightarrow 0,
\end{aligned}
$$

as $n \rightarrow \infty$. Thus, $y_{n} \rightarrow P_{\bigcap_{m=1}^{\infty}} c_{m}\left(u_{1}\right)$, as $n \rightarrow \infty$.

Since $y_{n+1}-y_{n} \rightarrow 0$ and $v_{n}=y_{n}+k_{n}\left(y_{n}-y_{n-1}\right)$, then $v_{n} \rightarrow P_{\cap_{m=1}^{\infty}} c_{m}\left(u_{1}\right)$, as $n \rightarrow \infty$.

Since $u_{n+1} \in Q_{n+1} \subset C_{n+1}$, then from (23), $\left\|w_{n}-u_{n+1}\right\|^{2} \leq \alpha_{n}\left\|y_{n}-u_{n+1}\right\|^{2}+\beta_{n} \| u_{n+1}-$ $u_{n}\left\|^{2}+\gamma_{n}\right\| e_{n}-u_{n+1}\left\|^{2}+k_{n}^{2}\right\| y_{n}-y_{n-1} \|^{2}-2 \alpha_{n} k_{n}\left\langle y_{n}-u_{n+1}, y_{n-1}-y_{n}\right\rangle \rightarrow 0$, as $n \rightarrow \infty$. Thus, $w_{n} \rightarrow$ $P_{\bigcap_{m=1}^{\infty} C_{m}}\left(u_{1}\right)$, as $n \rightarrow \infty$.

Step 8. $P_{\bigcap_{m=1}^{\infty}} c_{m}\left(u_{1}\right) \in \bigcap_{i=1}^{\infty}\left(A_{i}+B_{i}\right)^{-1} 0 \cap V I(K, T)$.

We shall first show that $P_{\bigcap_{m=1}^{\infty}} C_{m}\left(u_{1}\right) \in V I(K, T)$.

For this, define

$$
B v=\left\{\begin{array}{l}
T v+N_{K} v, \text { if } v \in K, \\
\varnothing, \text { if } v \bar{\in} K,
\end{array}\right.
$$

where $N_{K} v=\{w \in H:\langle v-u, w\rangle \geq 0, \forall u \in K\}$ is the normal cone to $K$ at $v \in K$. It is well-known that $B: H \rightarrow H$ is $\mathrm{m}$-accretive and $0 \in B v$ if and only if $v \in V I(K, T)$ [28].

Let $z \in B v=T v+N_{K} v$, then $z-T v \in N_{K} v$. From the definition of the normal cone, we have

$$
\left\langle v-y_{n}, z-T v\right\rangle \geq 0
$$

From Lemma 7, we have:

$$
\left\langle u_{n}-\lambda_{n} T u_{n}-P_{K}\left(u_{n}-\lambda_{n} T u_{n}\right), P_{K}\left(u_{n}-\lambda_{n} T u_{n}\right)-v\right\rangle \geq 0, \quad \forall v \in K,
$$

which implies that

$$
\left\langle v-P_{K}\left(u_{n}-\lambda_{n} T u_{n}\right), \frac{P_{K}\left(u_{n}-\lambda_{n} T u_{n}\right)-u_{n}}{\lambda_{n}}+T u_{n}\right\rangle \geq 0, \quad \forall v \in K .
$$


In view of (25) and (26), we know that

$$
\begin{aligned}
& \left\langle v-y_{n}, z\right\rangle \geq\left\langle v-y_{n}, T v\right\rangle \\
& =\left\langle v-P_{K}\left(u_{n}-\lambda_{n} T u_{n}\right), T v\right\rangle \\
& \geq\left\langle v-P_{K}\left(u_{n}-\lambda_{n} T u_{n}\right), T v\right\rangle-\left\langle v-P_{K}\left(u_{n}-\lambda_{n} T u_{n}\right), \frac{P_{K}\left(u_{n}-\lambda_{n} T u_{n}\right)-u_{n}}{\lambda_{n}}+T u_{n}\right\rangle \\
& =\left\langle v-P_{K}\left(u_{n}-\lambda_{n} T u_{n}\right), T v-T P_{K}\left(u_{n}-\lambda_{n} T u_{n}\right)\right\rangle \\
& +\left\langle v-P_{K}\left(u_{n}-\lambda_{n} T u_{n}\right), T P_{K}\left(u_{n}-\lambda_{n} T u_{n}\right)-T u_{n}\right\rangle \\
& -\left\langle v-P_{K}\left(u_{n}-\lambda_{n} T u_{n}\right), \frac{P_{K}\left(u_{n}-\lambda_{n} T u_{n}\right)-u_{n}}{\lambda_{n}}\right\rangle \\
& \geq\left\langle v-P_{K}\left(u_{n}-\lambda_{n} T u_{n}\right), T P_{K}\left(u_{n}-\lambda_{n} T u_{n}\right)-T u_{n}\right\rangle \\
& -\left\langle v-P_{K}\left(u_{n}-\lambda_{n} T u_{n}\right), \frac{P_{K}\left(u_{n}-\lambda_{n} T u_{n}\right)-u_{n}}{\lambda_{n}}\right\rangle \\
& =\left\langle v-y_{n}, T y_{n}-T u_{n}\right\rangle-\left\langle v-y_{n}, \frac{y_{n}-u_{n}}{\lambda_{n}}\right\rangle .
\end{aligned}
$$

Taking limit on both sides of the above inequality, we have: $\left\langle v-P_{\bigcap_{m=1}^{\infty}} C_{m}\left(u_{1}\right), z\right\rangle \geq 0$, which implies from the fact $B$ is m-accretive that $P_{\bigcap_{m=1}^{\infty} C_{m}}\left(u_{1}\right) \in B^{-1} 0$, and then $P_{\bigcap_{m=1}^{\infty} C_{m}}\left(u_{1}\right) \in V I(K, T)$.

Next, we shall show that $P_{\bigcap_{m=1}^{\infty} C_{m}}\left(u_{1}\right) \in \bigcap_{i=1}^{\infty}\left(A_{i}+B_{i}\right)^{-1} 0$.

In fact, if, otherwise, $P_{\bigcap_{m=1}^{\infty} C_{m}}\left(u_{1}\right) \bar{\in} \bigcap_{i=1}^{\infty}\left(A_{i}+B_{i}\right)^{-1} 0$. Then $P_{\bigcap_{m=1}^{\infty} c_{m}}\left(u_{1}\right) \neq \sum_{i=1}^{\infty} \omega_{n, i}(I+$ $\left.s_{n, i} B_{i}\right)^{-1}\left(I-s_{n, i} A_{i}\right) P_{\bigcap_{m=1}^{\infty} c_{m}}\left(u_{1}\right)$.

Since $w_{n}=\alpha_{n} v_{n}+\beta_{n} \sum_{i=1}^{\infty} \omega_{n, i}\left(I+s_{n, i} B_{i}\right)^{-1}\left(I-s_{n, i} A_{i}\right) P_{K}\left(u_{n}-\lambda_{n} T y_{n}\right)+\gamma_{n} e_{n}$, then $\beta_{n}\left[\sum_{i=1}^{\infty} \omega_{n, i}\left(I+s_{n, i} B_{i}\right)^{-1}\left(I-s_{n, i} A_{i}\right) P_{K}\left(u_{n}-\lambda_{n} T y_{n}\right)-w_{n}\right]=\alpha_{n}\left(w_{n}-v_{n}\right)+\gamma_{n}\left(w_{n}-e_{n}\right) \rightarrow 0$, as $n \rightarrow \infty$.

Since $i n f_{n} \beta_{n}>0$, then there exists a subsequence of $\{n\}$, which is still denoted by $\{n\}$ such that $\sum_{i=1}^{\infty} \omega_{n, i}\left(I+s_{n, i} B_{i}\right)^{-1}\left(I-s_{n, i} A_{i}\right) P_{K}\left(u_{n}-\lambda_{n} T y_{n}\right)-w_{n} \rightarrow 0$, as $n \rightarrow \infty$.

Thus, $\sum_{i=1}^{\infty} \omega_{n, i}\left(I+s_{n, i} B_{i}\right)^{-1}\left(I-s_{n, i} A_{i}\right) P_{K}\left(u_{n}-\lambda_{n} T y_{n}\right) \rightarrow P_{\bigcap_{m=1}^{\infty} c_{m}}\left(u_{1}\right)$, as $n \rightarrow \infty$. From Step 1 and $u_{n}-y_{n} \rightarrow 0$, we can also know that $\sum_{i=1}^{\infty} \omega_{n, i}\left(I+s_{n, i} B_{i}\right)^{-1}\left(I-s_{n, i} A_{i}\right) P_{K}\left(u_{n}-\lambda_{n} T u_{n}\right) \rightarrow$ $P_{\cap_{m=1}^{\infty} C_{m}}\left(u_{1}\right)$, as $n \rightarrow \infty$.

Since $H$ satisfies Opial's condition, $\lambda_{n} \rightarrow 0$ and $P_{\bigcap_{m=1}^{\infty} c_{m}}\left(u_{1}\right) \in K$, then

$$
\begin{aligned}
& \liminf _{n \rightarrow \infty}\left\|u_{n}-\lambda_{n} T u_{n}-P_{\cap_{m=1}^{\infty} c_{m}}\left(u_{1}\right)\right\| \\
& <\liminf _{n \rightarrow \infty}\left\|u_{n}-\lambda_{n} T u_{n}-\sum_{i=1}^{\infty} \omega_{n, i}\left(I+s_{n, i} B_{i}\right)^{-1}\left(I-s_{n, i} A_{i}\right) P_{\bigcap_{m=1}^{\infty} C_{m}}\left(u_{1}\right)\right\| \\
& \leq \liminf _{n \rightarrow \infty}\left\|u_{n}-\lambda_{n} T u_{n}-\sum_{i=1}^{\infty} \omega_{n, i}\left(I+s_{n, i} B_{i}\right)^{-1}\left(I-s_{n, i} A_{i}\right) P_{K}\left(u_{n}-\lambda_{n} T u_{n}\right)\right\| \\
& +\liminf _{n \rightarrow \infty} \| \sum_{i=1}^{\infty} \omega_{n, i}\left(I+s_{n, i} B_{i}\right)^{-1}\left(I-s_{n, i} A_{i}\right) P_{K}\left(u_{n}-\lambda_{n} T u_{n}\right) \\
& -\sum_{i=1}^{\infty} \omega_{n, i}\left(I+s_{n, i} B_{i}\right)^{-1}\left(I-s_{n, i} A_{i}\right) P_{\bigcap_{m=1}^{\infty} C_{m}}\left(u_{1}\right) \| \\
& \leq \liminf _{n \rightarrow \infty}\left\|u_{n}-\lambda_{n} T u_{n}-P_{\bigcap_{m=1}^{\infty} C_{m}}\left(u_{1}\right)\right\|,
\end{aligned}
$$

which makes a contradiction! Thus, $P_{\bigcap_{m=1}^{\infty}} C_{m}\left(u_{1}\right) \in \bigcap_{i=1}^{\infty}\left(A_{i}+B_{i}\right)^{-1} 0$.

Step 9. $P_{\bigcap_{m=1}^{\infty} C_{m}}\left(u_{1}\right)=P_{\bigcap_{i=1}^{\infty}\left(A_{i}+B_{i}\right)^{-1} 0 \cap V I(K, T)}\left(u_{1}\right)$.

From Step 8, $\left\|P_{\cap_{i=1}^{\infty}\left(A_{i}+B_{i}\right)^{-1} 0 \cap V I(K, T)}\left(u_{1}\right)-u_{1}\right\| \leq\left\|P_{\bigcap_{m=1}^{\infty} C_{m}}\left(u_{1}\right)-u_{1}\right\|$.

On the other hand, since $\bigcap_{i=1}^{\infty}\left(A_{i}+B_{i}\right)^{-1} 0 \bigcap V I(K, T) \subset \bigcap_{m=1}^{\infty} C_{m}$, then

$$
\left\|P_{\bigcap_{m=1}^{\infty} C_{m}}\left(u_{1}\right)-u_{1}\right\| \leq\left\|P_{\bigcap_{i=1}^{\infty}\left(A_{i}+B_{i}\right)^{-1} 0 \cap V I(K, T)}\left(u_{1}\right)-u_{1}\right\| .
$$

Thus

$$
\left\|P_{\bigcap_{i=1}^{\infty}\left(A_{i}+B_{i}\right)^{-1} 0 \cap V I(K, T)}\left(u_{1}\right)-u_{1}\right\|=\left\|P_{\bigcap_{m=1}^{\infty} C_{m}}\left(u_{1}\right)-u_{1}\right\| .
$$

Using Lemma 7, we have

$$
\begin{aligned}
& \left\|P_{\bigcap_{i=1}^{\infty}\left(A_{i}+B_{i}\right)^{-1} 0 \cap V I(K, T)}\left(u_{1}\right)-P_{\bigcap_{m=1}^{\infty} C_{m}}\left(u_{1}\right)\right\|^{2}+\left\|P_{\bigcap_{m=1}^{\infty} C_{m}}\left(u_{1}\right)-u_{1}\right\|^{2} \\
& \leq\left\|P_{\bigcap_{i=1}^{\infty}\left(A_{i}+B_{i}\right)^{-1} 0 \cap V I(K, T)}\left(u_{1}\right)-u_{1}\right\|^{2}=\left\|P_{\bigcap_{m=1}^{\infty} C_{m}}\left(u_{1}\right)-u_{1}\right\|^{2},
\end{aligned}
$$

which implies that $P_{\bigcap_{i=1}^{\infty}\left(A_{i}+B_{i}\right)^{-1} 0 \cap V I(K, T)}\left(u_{1}\right)=P_{\bigcap_{m=1}^{\infty} C_{m}}\left(u_{1}\right)$. 
This completes the proof.

2.3.2. The Second Kind Iteration Theorems

The following result is a special result of Lemma 10 in [19]:

Theorem 4. Let $H$ be a real Hilbert space and $K$ be a non-empty closed and convex subset of $H$. Suppose $f$ : $H \rightarrow H$ is a contraction with $k \in(0,1), F: H \rightarrow H$ is a strongly positive linear bounded operator with coefficient $\xi$ and $U: H \rightarrow H$ is non-expansive mapping. If $0<\lambda<\frac{\xi}{2 k}$, then there exists $x_{t}$ which satisfies $x_{t}=t \lambda f\left(x_{t}\right)+(I-t F) U x_{t}$, for $0<t \leq\|F\|^{-1}$. Moreover, $x_{t} \rightarrow p_{0}$ as $t \rightarrow 0$, and $p_{0}$ satisfies the following variational inequality: for $\forall z \in F i x(U)$,

$$
\left\langle(F-\lambda f) p_{0}, p_{0}-z\right\rangle \leq 0
$$

In Lemma 10 of [19], we can also know that the solution of the variational inequality (27) is unique.

Theorem 5. Under the assumptions of Theorem 2, $\left\{u_{n}\right\}$ generated by (16) converges strongly to $P_{\bigcap_{i=1}^{\infty}\left(A_{i}+B_{i}\right)^{-1} 0}\left(u_{1}\right)$. Set $\tilde{x}=P_{\bigcap_{i=1}^{\infty}\left(A_{i}+B_{i}\right)^{-1} 0}\left(u_{1}\right)$. If $\widetilde{x}=P_{\bigcap_{i=1}^{\infty}\left(A_{i}+B_{i}\right)^{-1} 0}[\lambda f(\widetilde{x})-F(\widetilde{x})+\widetilde{x}]$, then $\tilde{x}$ satisfies the following variational inequality: $\forall z \in \bigcap_{i=1}^{\infty}\left(A_{i}+B_{i}\right)^{-1} 0$,

$$
\langle(F-\lambda f) \widetilde{x}, \widetilde{x}-z\rangle \leq 0
$$

Proof. It follows from Lemma 7 that $\langle(F-\lambda f) \widetilde{x}, \widetilde{x}-z\rangle \leq 0, \forall z \in \bigcap_{i=1}^{\infty}\left(A_{i}+B_{i}\right)^{-1} 0$. Since Theorem 4 tells us that (28) has a unique solution, then we know that $\left\{u_{n}\right\}$ generated by (16) converges strongly to the unique solution of variational inequality (28).

Remark 5. The assumption that $\widetilde{x}=P_{\bigcap_{i=1}^{\infty}\left(A_{i}+B_{i}\right)^{-1} 0}[\lambda f(\widetilde{x})-F(\widetilde{x})+\widetilde{x}]$ is reasonable. For example, we may take $f(x)=\frac{x}{2}$ and $F(x)=\frac{\lambda x}{2}$, for $x \in H$.

Remark 6. For projection iterative algorithms such as (16), rare work can be found to show that the limit of the iterative sequences is also the solution of a kind of variational inequalities.

\section{Applications}

\subsection{Preparation for Discussion of Capillarity Systems}

To present some examples in this section, we need some basic definitions in Banach spaces.

Let $E$ be a real Banach space with $E^{*}$ being its dual space and let $\langle\cdot, \cdot\rangle$ denote the generalized duality pairing between $E$ and $E^{*}$.

Definition 4. (see [29]) Recall that $J: E \rightarrow 2^{E^{*}}$ is called the normalized duality mapping if $\forall x \in E$,

$$
J x=\left\{f \in E^{*}:\langle x, f\rangle=\|x\|^{2}=\|f\|^{2}\right\} .
$$

Definition 5. (see [29]) Recall that $\mathcal{A}: E \rightarrow E^{*}$ is said to be a monotone mapping if $\forall x_{i} \in D(\mathcal{A}), i=1,2$, one has

$$
\left\langle x_{1}-x_{2}, \mathcal{A} x_{1}-\mathcal{A} x_{2}\right\rangle \geq 0 \text {. }
$$

A monotone mapping $\mathcal{A}: E \rightarrow E^{*}$ is said to be maximal monotone if $R(J+r \mathcal{A})=E^{*}, \forall r>0$.

Definition 6. (see [29]) Recall that a mapping $\mathcal{B}: E \rightarrow E^{*}$ is said to be coercive if $\left\{x_{n}\right\} \subset D(\mathcal{B})$ with $\lim _{n \rightarrow \infty}\left\|x_{n}\right\|=+\infty$, then $\lim _{n \rightarrow \infty} \frac{\left\langle x_{n}, \mathcal{B} x_{n}\right\rangle}{\left\|x_{n}\right\|}=+\infty$. 
Definition 7. (see [29]) Recall that a mapping $\mathcal{B}: D(\mathcal{B})=E \rightarrow E^{*}$ is said to be a hemi-continuous mapping if $\mathcal{B}(x+t y) \rightarrow \mathcal{B} x$, as $t \rightarrow 0$, for $\forall x, y \in E$.

Definition 8. (see [29]) $\psi: E \rightarrow(-\infty,+\infty]$ is said to be a proper convex functional if there exists $u_{0} \in E$ such that $\psi\left(u_{0}\right)<+\infty$ and $\psi((1-\lambda) u+\lambda v) \leq(1-\lambda) \psi(u)+\lambda \psi(v)$, for $\forall u, v \in E$ and $\lambda \in[0,1] . \psi$ : $E \rightarrow(-\infty,+\infty]$ is said to be lower-semi-continuous: $\liminf _{y \rightarrow x} \psi(y) \geq \psi(x)$, for $\forall x \in E$. The subdifferential of $\psi, \partial \psi: E \rightarrow E^{*}$, is defined by:

$$
\partial \psi(u)=\left\{w \in E^{*}: \psi(u)-\psi(v) \leq\langle u-v, w\rangle, \quad \forall v \in E\right\}, \quad \forall u \in E .
$$

\subsection{Applications to Capillarity Elliptic Systems}

Example 1. Suppose $\Omega$ is bounded conical domain in $R^{n}(n \in \mathbb{N})$ with $\Gamma \in C^{1}, \vartheta$ is the exterior normal derivative of $\Gamma, \varepsilon_{i}$ is a non-negative constant, $\lambda_{i}$ is a positive number, $f_{i}(x) \in L^{p_{i}}(\Omega)$ is a given function, for $i=1,2, \cdots, M$. In addition, $\beta_{x}: R \rightarrow R$ is the subdifferential of $\varphi_{x}$, where $\varphi_{x}=\varphi(x, \cdot): R \rightarrow R$, for each $x \in \Gamma$. Suppose $\frac{2 n}{n+1}<p_{i}<+\infty, i=1,2, \cdots$, M. If $p_{i} \geq n$ then $1 \leq q_{i}, r_{i}<+\infty$ and if $p_{i}<n$ then $1 \leq q_{i}, r_{i} \leq \frac{n p_{i}}{n-p_{i}}$ for $i=1,2, \cdots, M$.

The following capillarity system is studied in [30]:

$$
\left\{\begin{array}{l}
-\operatorname{div}\left[\left(1+\frac{\mid \nabla u^{(i) \mid p_{i}}}{\sqrt{1+\left|\nabla u^{(i)}\right|^{2 p_{i}}}}\right)\left|\nabla u^{(i)}\right| p_{i}-2 \nabla u^{(i)}\right]+\lambda_{i}\left(\left|u^{(i)}\right| q^{q_{i}-2} u^{(i)}+\left|u^{(i)}\right|^{r_{i}-2} u^{(i)}\right) \\
+\varepsilon_{i} g_{i}\left(x, \nabla u^{(i)}, u^{(i)}\right)=f_{i}(x), \quad x \in \Omega \\
-<\vartheta,\left(1+\frac{\left|\nabla u^{(i)}\right| p_{i}}{\sqrt{1+\left|\nabla u^{(i)}\right|^{2 p_{i}}}}\right)\left|\nabla u^{(i)}\right|^{p_{i}-2} \nabla u^{(i)}>\in \beta_{x}\left(u^{(i)}(x)\right), \quad x \in \Gamma, \quad i=1,2, \cdots, M,
\end{array}\right.
$$

where $|\cdot|$ and $<\cdot, \cdot>$ denote the norm and inner product in $R^{n}$, respectively.

The study on (29) in [30] is based on the following assumptions.

(1) $\forall x \in \Gamma, \varphi_{x}=\varphi(x, \cdot): R \rightarrow R$ is a proper convex and lower-semi-continuous mapping with $\varphi_{x}(0)=0$.

(2) $0 \in \beta_{x}(0), \forall t \in R, x \in \Gamma \rightarrow\left(I+\lambda \beta_{x}\right)^{-1}(t) \in R$ is measurable for $\lambda>0$.

(3) For each $i \in\{1,2, \cdots, M\}, g_{i}: \Omega \times R^{n} \times R \rightarrow R$ satisfies Caratheodory's conditions and satisfies that

$$
\begin{aligned}
& \qquad\left|g_{i}\left(x, r_{1}, r_{2}, \cdots, r_{n+1}\right)-g_{i}\left(x, s_{1}, s_{2}, \cdots, s_{n+1}\right)\right| \leq b_{i}\left|r_{n+1}-s_{n+1}\right|, \\
& \text { for } \forall\left(r_{1}, r_{2}, \cdots, r_{n+1}\right),\left(s_{1}, s_{2}, \cdots, s_{n+1}\right) \in R^{n+1} .
\end{aligned}
$$

By using splitting method, the sufficient condition that (29) has non-trivial solution is obtained:

Theorem 6. (see [30]) If $u^{(i)} \in L^{p_{i}}(\Omega)$ satisfies that

$$
\int_{\Omega}\left[g_{i}\left(x, \nabla u^{(i)}, u^{(i)}\right)-f_{i}\right]\left|u^{(i)}\right|^{p_{i}-2} u^{(i)} d x \geq 0,
$$

for $i=1,2, \cdots, M$, then $u=\left(u^{(1)}, u^{(2)}, \ldots, u^{(M)}\right)$ is the non-trivial solution of capillarity system (29).

Based on Example 1, we present the following example:

Example 2. Suppose $\Omega, \Gamma$ and $\vartheta$ are the same as those in Example 1. Suppose $\lambda_{i}>0, \frac{2 n}{n+1}<p_{i}<+\infty$. If $p_{i} \geq n$, then suppose $1 \leq q_{i}, r_{i}<+\infty$ and if $p_{i}<n$, then suppose $1 \leq q_{i}, r_{i} \leq \frac{n p_{i}}{n-p_{i}}$, for $i \in \mathbb{N}$. 
Now, we will discuss the following capillarity systems.

$$
\left\{\begin{array}{l}
-\operatorname{div}\left[\left(1+\frac{\mid \nabla u^{(i) \mid p_{i}}}{\sqrt{1+\left|\nabla u^{(i)}\right|^{2 p_{i}}}}\right)\left|\nabla u^{(i)}\right|^{p_{i}-2} \nabla u^{(i)}\right] \\
+\lambda_{i}\left(\left|u^{(i)}\right| q^{q_{i}-2} u^{(i)}+\left.\left|u^{(i)}\right|\right|^{r_{i}-2} u^{(i)}\right)+u^{(i)}(x)=f_{i}(x), \quad x \in \Omega \\
-<\vartheta,\left(1+\frac{\left|\nabla u^{(i)}\right|_{i}}{\sqrt{1+\left|\nabla u^{(i)}\right|^{2 p_{i}}}}\right)\left|\nabla u^{(i)}\right| p_{i}-2 \nabla u^{(i)}>=0, \quad x \in \Gamma, \quad i \in \mathbb{N} .
\end{array}\right.
$$

Please note that (30) is the extension from the finite case of (29) to that for infinite case. However, both the capillarity equations and the boundary conditions are the special case of (29) in the sense that $\varepsilon_{i} \equiv 1$ and $g_{i}\left(x, \nabla u^{(i)}, u^{(i)}\right) \equiv u^{(i)}$ for $i \in \mathbb{N}$ and $\beta_{x} \equiv 0$, for $x \in \Gamma$.

Lemma 9. (see [30]) The mapping $U_{i}: W^{1, p_{i}}(\Omega) \rightarrow\left(W^{1, p_{i}}(\Omega)\right)^{*}$ defined by

$$
\begin{gathered}
\left\langle w, U_{i} u\right\rangle=\int_{\Omega}<\left(1+\frac{|\nabla u|^{p_{i}}}{\sqrt{1+|\nabla u|^{2 p_{i}}}}\right)|\nabla u|^{p_{i}-2} \nabla u, \\
\nabla v>d x+\lambda_{i} \int_{\Omega}|u(x)|^{q_{i}-2} u(x) v(x) d x+\lambda_{i} \int_{\Omega}|u(x)|^{r_{i}-2} u(x) v(x) d x,
\end{gathered}
$$

for $\forall u, w \in W^{1, p_{i}}(\Omega)$, is everywhere defined, hemi-continuous, monotone and coercive, for each $i \in \mathbb{N}$.

Lemma 10. (see [30]) Define $B_{i}: L^{2}(\Omega) \rightarrow L^{2}(\Omega)$ by

$$
D\left(B_{i}\right)=\left\{u \in L^{2}(\Omega) \mid \exists f \in L^{2}(\Omega) \text { such that } f \in U_{i} u\right\} .
$$

For $u \in D\left(B_{i}\right), B_{i} u=\left\{f \in L^{2}(\Omega) \mid f \in U_{i} u\right\}$. Then $B_{i}: L^{2}(\Omega) \rightarrow L^{2}(\Omega)$ is m-accretive, for each $i \in \mathbb{N}$.

Lemma 11. (see [30]) The mapping $A_{i}: D\left(A_{i}\right) \subset L^{2}(\Omega) \rightarrow L^{2}(\Omega)$ defined by

$$
\left(A_{i} u\right)(x)=u(x)-f_{i}(x), \quad \forall u(x) \in D\left(A_{i}\right),
$$

is $\mu_{i}$-inversely strongly accretive, for $\mu_{i} \in(0,1]$, for $i \in \mathbb{N}$.

Theorem 7. If $f_{i}(x) \equiv \lambda_{i}\left(|k|^{q_{i}-1}+|k|^{r_{i}-1}\right) \operatorname{sgn} k+k$, then $\left\{u^{(i)} \equiv k: i \in \mathbb{N}\right\}$ is the solution of capillarity system (30). Moreover, $\{k\}=\bigcap_{i=1}^{\infty}\left(A_{i}+B_{i}\right)^{-1} 0$.

Proof. It is easy to see that $\left\{u^{(i)} \equiv k: i \in \mathbb{N}\right\}$ is the solution of capillarity system (30) and $\{k\} \subset$ $\bigcap_{i=1}^{\infty}\left(A_{i}+B_{i}\right)^{-1} 0$. Now, we shall show that $\{k\} \supset \bigcap_{i=1}^{\infty}\left(A_{i}+B_{i}\right)^{-1} 0$.

In fact, if $A_{i} u+B_{i} u=0$ and $A_{i} v+B_{i} v=0$, then $u+B_{i} u=v+B_{i} v$, which implies that

$$
0 \leq\left\langle u-v, B_{i} u-B_{i} v\right\rangle=\langle u-v, v-u\rangle \leq 0 .
$$

Thus, $u=v$ and then $\bigcap_{i=1}^{\infty}\left(A_{i}+B_{i}\right)^{-1} 0$ is a singleton. Since $k \in \bigcap_{i=1}^{\infty}\left(A_{i}+B_{i}\right)^{-1} 0$, then the result follows.

Theorem 8. Let $f_{i}(x) \equiv \lambda_{i}\left(|k|^{q_{i}-1}+|k|^{r_{i}-1}\right) \operatorname{sgn} k+k$, for $i \in \mathbb{N}$. Suppose $A_{i}$ and $B_{i}$ are the same as those in Lemmas 10 and 11, respectively. Let $F: L^{2}(\Omega) \rightarrow L^{2}(\Omega)$ be any strongly positive linear bounded operator with coefficient $\xi>0$ and $f: L^{2}(\Omega) \rightarrow L^{2}(\Omega)$ be a contraction with coefficient $k \in(0,1)$. Constructing the following iterative algorithm: 


$$
\left\{\begin{array}{l}
u_{0}, u_{1} \in L^{2}(\Omega) \text { chosen arbitrarily, } e_{1} \in L^{2}(\Omega) \\
z_{0}=u_{0} \\
z_{n}=\delta_{n} \lambda f\left(u_{n}\right)+\left(I-\delta_{n} F\right) u_{n}, \\
v_{n}=z_{n}+k_{n}\left(z_{n}-z_{n-1}\right) \\
w_{n}=\alpha_{n} v_{n}+\beta_{n} \sum_{i=1}^{\infty} \omega_{n, i}\left(I+s_{n, i} B_{i}\right)^{-1}\left(I-s_{n, i} A_{i}\right)\left(\frac{v_{n}+w_{n}}{2}\right)+\gamma_{n} e_{n}, \\
C_{1}=L^{2}(\Omega)=Q_{1} \\
\quad C_{n+1}=\left\{p \in C_{n}:\left\|w_{n}-p\right\|^{2} \leq \frac{2 \alpha_{n}+\beta_{n}}{2-\beta_{n}}\left\|z_{n}-p\right\|^{2}+\frac{2 \gamma_{n}}{2-\beta_{n}}\left\|e_{n}-p\right\|^{2}\right. \\
\left.\quad \quad+\frac{2 \alpha_{n}+\beta_{n}}{2-\beta_{n}} k_{n}^{2}\left\|z_{n}-z_{n-1}\right\|^{2}-2 k_{n} \frac{2 \alpha_{n}+\beta_{n}}{2-\beta_{n}}\left\langle z_{n}-p, z_{n-1}-z_{n}\right\rangle\right\} \\
\quad Q_{n+1}=\left\{p \in C_{n+1}:\left\|u_{1}-p\right\|^{2} \leq\left\|P_{C_{n+1}}\left(u_{1}\right)-u_{1}\right\|^{2}+\sigma_{n+1}\right\} \\
u_{n+1} \in Q_{n+1}, \quad n \in \mathbb{N} .
\end{array}\right.
$$

Under the assumptions of Theorem 2 , using the result of Theorem 5 , one has $u_{n}(x) \rightarrow P_{\bigcap_{i=1}^{\infty}\left(A_{i}+B_{i}\right)^{-1} 0}\left(u_{1}\right)$, which is the unique solution of capillarity system (30) and satisfies the following variational inequality: For $\forall z(x) \in \bigcap_{i=1}^{\infty}\left(A_{i}+B_{i}\right)^{-1} 0$,

$$
\left.\left\langle(F-\lambda f) P_{\bigcap_{i=1}^{\infty}\left(A_{i}+B_{i}\right)^{-1} 0}\left(u_{1}\right), P_{\bigcap_{i=1}^{\infty}\left(A_{i}+B_{i}\right)^{-1} 0}\left(u_{1}\right)-z\right)\right\rangle \leq 0 .
$$

Remark 7. From Theorem 8 we can easily see the relationship among the solution of capillarity system, the solution of variational inequality and the zero of sum of infinitely many m-accretive mappings and infinitely many $\mu_{i}$-inversely strongly accretive mappings.

Author Contributions: L.W. and R.P.A. contributed are equally to Sections 1 and 2. The Y.S. contributed to Section 3.

Funding: Supported by the National Natural Science Foundation of China (11071053), Natural Science Foundation of Hebei Province (A2014207010), Key Project of Science and Research of Hebei Educational Department (ZD2019073), Key Project of Science and Research of Hebei University of Economics and Business (2018ZD06), Youth Project of Science and Research of Hebei University of Economics and Business (2017KYQ09) and Youth Project of Science and Research of Hebei Educational Department (QN2017328).

Acknowledgments: Thanks for the reviewers' valuable opinions and careful work.

Conflicts of Interest: The authors declare that they have no competing interests.

\section{References}

1. Opial, Z. Weak convergence of the sequence of successive approximations for nonexpansive mappings. Bull. Am. Math. Soc. 1967, 73, 591-597. [CrossRef]

2. Takahashi, W. Nonlinear Functional Analysis. Fixed Point Theory and its Applications; Yokohama Publishers: Yokohama, Japan, 2000

3. Wei, L.; Shi, A.F. Splitting-midpoint method for zeros of the sum of accretive operator and $\mu$-inversely strongly accretive operator in a q-uniformly smooth Banach space and its applications. J. Inequal. Appl. 2015, 2015, 183. [CrossRef]

4. Shehu, Y. Iterative approximations for zeros of the sum of accretive operators in Banach spaces. J. Funct. Spaces 2016, 2016, 5973468. [CrossRef]

5. Combettes, P.L.; Wajs, V.R. Signal recovery by proximal forward-backward splitting. Multiscale Model. Simul. 2005, 4, 1168-1200. [CrossRef]

6. Tseng, P. A mordified forward-backward splitting method for maximal monotone mappings. SIAM J. Control Optim. 2000, 38, 431-446. [CrossRef]

7. Khan, S.A.; Suantai, S.; Cholamjiak, W. Shrinking projection methods involving inertial forward-backward splitting methods for inclusion problems. RACSAM 2018, 113, 645-656. [CrossRef]

8. Moudafi, A.; Oliny, M. Convergence of a splitting inertial proximal method for monotone operators. J. Comput. Appl. Math. 2003, 155, 447-454. [CrossRef]

9. Lorenz, D.; Pock, T. An inertial forward-backward algorithm for monotone inclusions. J. Math. Imaging Vis. 2015, 51, 311-325. [CrossRef] 
10. Cholamjiak, P. A generalized forward-backward splitting method for solving quasi inclusion problems in Banach spaces. Numer. Algorithm 2016, 71, 915-932. [CrossRef]

11. Lopez G.; Martin-Marquez, V.; Wang, F.; Xu, H.K. Forward-backward splitting method for accretive operators in Banach spaces. Abstr. Appl. Anal. 2012, 2012, 109236. [CrossRef]

12. Qin, X.; Cho, S.Y.; Wang, L. Convergence of splitting algorithms for the sum of two accretive operators with applications. Fixed Point Theory Appl. 2014, 2014, 166. [CrossRef]

13. Bot, R.I.; Csetnek, E.R.; Hendrich, C. Inertial Douglas-Rachford splitting for monotone inclusion. Appl. Math. Comput. 2015, 256, 472-487.

14. Dong, Q.L.; Jiang, D.; Cholamjiak, P.; Shehu, Y. A strong convergence result involving an inertial forward-backward algorithm for monotone inlusions. J. Fixed Point Theory Appl. 2017, 19, 3097-3118. [CrossRef]

15. Wei, L.; Duan, L.L. A new iterative algorithm for the sum of two different types of finitely many accretive operators in Banach and its connection with capillarity equation. Fixed Point Theory Appl. 2015, 2015, 25. [CrossRef]

16. Wei, L.; Duan, L.L.; Agarwal, R.P.; Chen, R.; Zheng, Y.Q. Mordified forward-backward splitting midpoint method with superposition perturbations for sum of two kind of infinite accretive mappings and its applications. J. Inequal. Appl. 2017, 2017, 227. [CrossRef] [PubMed]

17. Wei, L.; Shen, Y.W.; Zheng, Y.Q.; Tan, R.L. A new iterative scheme for the sum of infinite m-accretive mappings and inversely strongly accretive mappings and its application. J. Nonlinear Var. Appl. 2017, 1, 345-356.

18. Wei, L.; Agarwal, R.P. Simple form of a projection set in hybrid iterative schemes for non-linear mappings, application of inequalities and computational experiments. J. Inequal. Anal. 2018, 2018, 179. [CrossRef] [PubMed]

19. Wei, L.; Agarwal, R.P. A new iterative algorithm for the sum of infinite $m$-accretive mappings and infinite $\mu_{i}$-inversely strongly accretive mappings and its applications to integro-differentail systems. Fixed Point Theory Appl. 2016, 2016, 7. [CrossRef]

20. Mosco, U. Convergence of convex sets and of solutions of variational inequalities. Adv. Math. 1969, 3, 510-585. [CrossRef]

21. Tsukada, M. Convergence of best approximations in a smooth Banach space. J. Approx. Theory 1984, 40, 301-309. [CrossRef]

22. Bruck, R.E. Properties of fixed-point sets of nonexpansive mappings in Banach spaces. Trans. Am. Math Soc. 1973, 179, 251-262. [CrossRef]

23. Xu, H.K. Inequalities in Banach space with applications. Nonlinear Anal. 1991, 16, 1127-1138. [CrossRef]

24. Nakajo, K.; Takahashi, W. Strong convergence theorems for nonexpansive mappings and nonexpansive semigroups. J. Math. Anal. Appl. 2003, 279, 327-379. [CrossRef]

25. Agarwal, R.P.; O'Regan, D.; Sahu, D.R. Fixed Point Theory for Lipschtz-type Mappings with Applications; Springer: New York, NY, USA, 2009

26. He, S.N.; Liu, L.L.; Gibali, A. Self-adaptive iterative method for solving boundedly Lipschitz continuous and strongly monotone variational inequalities. J. Inequal. Appl. 2018, 2018, 350. [CrossRef] [PubMed]

27. Gibali, A.; Reich, S.; Zalas, R. Ooter approximation methods for solving variational inequalities in Hilbert space. Optimization 2017, 66, 417-437. [CrossRef]

28. Rockafellar, R.T. On the maximality of sums of nonlinear monotone operators. Trans. Am. Math. Soc. 1970, 149, 75-88. [CrossRef]

29. Pascali, D.; Sburlan, S. Nonlinear Mappings of Monotone Type; Sijthoff Noordhoff International Publishers: Bucharest, Romania, 1978.

30. Wei, L.; Chen, R. Study on the existence of non-trivial solution of one kind capillarity systems. J. Math. (PRC) 2017, 37, 390-400. (In Chinese)

(C) 2019 by the authors. Licensee MDPI, Basel, Switzerland. This article is an open access article distributed under the terms and conditions of the Creative Commons Attribution (CC BY) license (http://creativecommons.org/licenses/by/4.0/). 